\title{
ADAR2 mediated Q/R editing of GluK2 regulates homeostatic plasticity of kainate receptors
}

Sonam Gurung, Ashley J. Evans, Kevin A. Wilkinson and Jeremy M. Henley*

School of Biochemistry, Centre for Synaptic Plasticity, Biomedical Sciences Building, University of Bristol, Bristol, BS8 1TD, UK

*please address correspondence to j.m.henley@bristol.ac.uk (Jeremy Henley) 


\begin{abstract}
Kainate receptors (KARs) are heteromeric glutamate-gated ion channels that regulate neuronal excitability and network function in the brain. Most KARs contain the subunit GluK2 and the precise properties of these GluK2-containing KARs are determined by additional factors including ADAR2mediated mRNA editing of a single codon that changes a genomically encoded glutamine $(\mathrm{Q})$ to arginine $(R)$ in the pore-lining region of GluK2. ADAR2-dependent $Q / R$ editing of GluK2 is dynamically regulated during homeostatic plasticity (scaling) elicited by suppression of synaptic activity with TTX. Here we show that TTX decreases levels of ADAR2 by enhancing its proteasomal degradation. This selectively reduces the numbers of GluK2 subunits that are edited and increases the surface expression of GluK2-containing KARs. Furthermore, we show that partial ADAR2 knockdown phenocopies and occludes TTX-induced scaling of KARs. These data indicate that activity-dependent regulation of ADAR2 proteostasis and GluK2 Q/R editing provides a mechanism for KAR homeostatic plasticity.
\end{abstract}

\title{
Keywords
}

Synapse, Kainate receptor, AMPA receptor, GluK2, ADAR2, mRNA editing, homeostatic plasticity, scaling. 
bioRxiv preprint doi: https://doi.org/10.1101/308650; this version posted April 25, 2018. The copyright holder for this preprint (which was not certified by peer review) is the author/funder, who has granted bioRxiv a license to display the preprint in perpetuity. It is made available under aCC-BY-NC-ND 4.0 International license.

\section{Introduction}

Kainate receptors (KARs) are ionotropic glutamate receptors that are tetrameric assemblies composed of combinations of five receptor subunits, GluK1-5 $(1,2)$. KARs can be located pre-, postand/or extrasynaptically, where they contribute to neurotransmitter release, postsynaptic depolarisation and the regulation of neuronal excitability (3). The variety of possible subunit combinations and co-assembly with Neto auxiliary subunits (4), creates a wide range of possible KAR subtypes, with receptors containing GluK2 and GluK5 being the most abundant (3).

Additional diversity of GluK2 containing KARs arises from RNA editing of GluK2 $(5,6)$ by the nuclear ADAR2 enzyme which edits pre-mRNAs encoding GluK2 and GluK1, as well as the AMPAR subunit GluA2, and other non-coding RNAs $(7,8)$. ADAR2 mediated $Q / R$ editing in the pore-lining region of GluK2 alters a genomically encoded glutamine residue to an arginine, changing both the calcium permeability and biophysical properties of the KARs. More specifically, GluK2(R) shows markedly reduced tetramerisation leading to its accumulation in the ER (9). Furthermore, assembled GluK2(R)-containing KARs that do reach the plasma membrane are calcium impermeable and have a channel conductance of less than $1 \%$ of non-edited GluK2(Q)-containing KARs (10).

ADAR2 levels are very low during embryogenesis but increase in the first postnatal week (11) to edit $\sim 80 \%$ of GluK2, $\sim 40 \%$ of GluK1 and $\sim 99 \%$ of GluA2 subunits in the mature brain (12-14). ADAR2 knockout mice die at the early postnatal stage, but can be rescued by expressing the edited form of GluA2, demonstrating that unedited AMPARs are fatally excitotoxic (15). In contrast, mice specifically deficient in GluK2 Q/R editing are viable but are seizure prone and adults display an aberrant form of NMDAR-independent long term potentiation (LTP) (16), demonstrating the importance of GluK2 editing to network function and synaptic plasticity.

NMDARs, AMPARs and KARs have all been shown to play roles in synaptic plasticity and their dysregulation is a prominent feature of cognitive decline in aging and neurodegenerative diseases (17). LTP and LTD (long-term depression) enhance or decrease the efficiency of synaptic transmission, respectively, and are primarily mediated by changes in the number and properties of AMPARs at the postsynaptic membrane. 'Classical' LTP requires the activation of NMDARs $(18,19)$. However, recent data from our lab has demonstrated a novel role for KARs as critical inducers of one form of LTP (KAR-LTPAMPAR) (20). Moreover, in addition to directly inducing synaptic plasticity of AMPARs, KARs themselves are plastic and undergo LTD (21) and LTP (22-24).

The assembly and progression of GluK2-containing KARs through the secretory pathway is controlled by a series of activity regulated checkpoints (25). Furthermore, chronic suppression of synaptic activity with TTX 'up-scales' KAR surface expression (25). Homeostatic plasticity (scaling) of AMPARs and NMDARs is essential for normal neuronal network and brain function because it 
constrains neuronal firing to within a tunable physiological range (26-28). The discovery of KAR scaling is important because regulation of the number of surface expressed KARs will be a critical factor for induction of KAR-LTPAMPAR, and therefore constitutes a key regulator of synaptic transmission and neuronal excitability. However, how KAR scaling occurs mechanistically is unclear.

Here we show that chronic suppression of network activity with TTX leads to the proteosomal degradation of ADAR2. This, in turn, reduces editing of GluK2 pre-mRNA with a consequent increase in unedited GluK2, thus enhancing homomeric KAR assembly, ER release and surface expression. Importantly, this up-scaling mechanism is specific to GluK2 as TTX did not change the editing status of GluA2. Together these data demonstrate a novel and selective role of mRNA editing by ADAR2 in homeostatic plasticity of KARs.

\section{Results}

Chronic suppression of network activity with TTX increases surface levels of GluK2-containing KARs

Chronic TTX treatment increases AMPAR surface expression and AMPAR excitatory postsynaptic currents (EPSCs) (reviewed in (29)). Surface levels of GluK2 containing KARs also upscale in response to TTX treatment (25). Consistent with this, we show robust scaling of both KAR (Figure $1 A, B$ ) and AMPAR subunits (Figure $1 A, C$ ). In contrast, epidermal growth factor receptor (EGFR), which is widely expressed at synapses (30), was not affected by TTX (Figure 1A,D). Intriguingly, TTX-mediated suppression of synaptic activity treatment also reduced GluK2 Q/R editing (Figure $1 E, F)$ but did not affect $Q / R$ editing of the AMPAR subunit GluA2 (SI Figure 1A,B,C). These findings suggest that KAR, but not AMPAR, up-scaling by TTX treatment may be mediated by decreased levels of GluK2 Q/R editing.

\section{Chronic suppression of network activity with TTX decreases ADAR2 levels}

We next tested the effects of chronic suppression of network activity on ADAR2 levels. Incubation with TTX for $24 \mathrm{~h}$ decreased ADAR2 levels by $~ 50 \%$, but ADAR1 levels were unaffected (Figure 2AD). Interestingly, longer periods of TTX treatment did not decrease ADAR2 levels any further (Figure $2 E, F)$, suggesting that a basal level of ADAR2 is retained even under long-term suppression of synaptic activity. As expected, the decrease in ADAR2 following $24 \mathrm{~h}$ TTX treatment occurs in the nucleus (Figure 2G,H), where ADAR2 binds to the GluK2 pre-mRNA prior to mRNA splicing and maturation (31). Moreover, both the intensity of ADAR2 signal and the percentage of ADAR2 expressing cells decreased significantly following $24 \mathrm{~h}$ TTX treatment (Figure $2 \mathrm{I}, \mathrm{J}, \mathrm{K}$ ).

\section{Complete and partial ADAR2 knockdown differentially alter GluK2 and GluA2 Q/R editing}


To further examine the role of ADAR2 in KAR scaling we generated two different shRNAs against ADAR2. One shRNA we named shRNA'complete ablated essentially all ADAR2 whereas another shRNA that we called shRNA 'Partial', reduced ADAR2 levels to $\sim 50 \%$ of the control (Figure $3 A, B$ ), comparable to the loss observed with TTX. Furthermore, shRNA Partial knockdown reduced the intensity of signal and percentage of cells expressing ADAR2 to levels similar to those elicited by TTX treatment (Figure 3C,D,E).

We next compared how ADAR2 knockdown affected GluK2 and GluA2 editing. As expected, shRNA 'complete' ADAR2 knockdown reduced GluK2 Q/R editing by over 60\%, whereas shRNA'Partial' knockdown of ADAR2 only reduced GluK2 Q/R editing by $\sim 20 \%$ (Figure 4A,B). DNA sequencing chromatographs from cDNA of cells treated with shRNA'Complete' show a dramatic change in the base read of the editing site to CAG $(Q)$ rather than CGG $(R)$, whereas neurons treated with shRNA'Partial' show a mixture of both CGG and CAG (Figure 4C). Interestingly, shRNA'Partial' knockdown of ADAR2 had no effect on the $Q / R$ editing of the AMPAR subunit GluA2 while the shRNA'complete' knockdown only reduced GluA2 editing by $\sim 30 \%$ (Figure $4 \mathrm{D}, \mathrm{E}, \mathrm{F}$ ). These data indicate that GluK2 editing levels are uniquely sensitive to ADAR2 changes and support a model whereby loss of ADAR2 during homeostatic scaling directly promotes surface expression of GluK2 containing KARs through a reduction in GluK2 editing.

\section{Partial ADAR2 knockdown phenocopies and occludes TTX upscaling of GluK2 containing KARs}

Intriguingly, shRNA 'Partial' reduces ADAR2 to levels comparable to those following TTX treatment and results in a similar shift in the proportions of edited to unedited GluK2. We therefore wondered if shRNA'Partial' alone is sufficient to mediate KAR upscaling. Partial ADAR2 knockdown significantly increased GluK2 surface expression with no effect on EGFR surface expression (Figure 5A,B,C), indicating that reduced levels of ADAR2 result in upscaling GluK2 containing KARs in the absence of TTX treatment. TTX treatment in combination with shRNA'Partial' knockdown was not additive (Figure 5A,B) consistent with TTX induced upscaling being mediated by a reduction in ADAR2 levels. In shRNA'Partial' ADAR2 knockdown neurons, $24 \mathrm{~h}$ TTX treatment did elicit a further decrease in ADAR2 levels (SI Figure 2A.B), indicating that even when already depleted, ADAR2 levels are still subject to activity-dependent regulation. However, despite the summative decrease in ADAR2 levels, shRNA 'Partial' ADAR2 knockdown combined with TTX treatment did not further decrease GluK2 Q/R editing compared to either treatment alone (Figure $5 D, E$ ). These data indicate that loss of ADAR2 is sufficient to mediate TTX induced up-scaling of KARs.

\section{Loss of ADAR2 during scaling is not dependent on its interaction with Pin1}

The nuclear protein Pin1 retains ADAR2 in the nucleus and interfering with this allows ADAR2 export to the cytosol, its ubiquitination and degradation (32). Our RT-qPCR experiments show that total 
ADAR2 mRNA levels are unaffected by TTX, indicating that scaling is not transcriptionally mediated (SI Figure 3). We therefore wondered if destabilising the Pin1-ADAR2 protein interaction underpins ADAR2 loss by TTX but Pin1 levels were unchanged following TTX treatment (Figure 6A,B).

We next investigated the role of ADAR2 phosphorylation at threonine 32 (T32) in regulating ADAR2 levels during scaling, since this has been reported to crucial for the ADAR2-Pin1 interaction (32). In contrast to WT and phosphonull (T32A) ADAR2, the phosphomimetic (T32D) ADAR2 mutant binds very strongly to Pin1 in GFP-trap assays (Figure 6C,D). We therefore tested if the phosphonull or phosphomimetic ADAR2 mutants were more sensitive to TTX treatment. We first knocked down endogenous ADAR2 and replaced it with HA-tagged WT ADAR2 (SI Figure 4A,B). More than 80\% of the cells expressed the knockdown-rescue, similar to the percentage of untreated neurons that express ADAR2 ( $S$ I Figure 4A,B). We then investigated the stability of the phosphonull or phosphomimetic ADAR2 mutants in response to TTX treatment. Similar to WT ADAR2, levels of both mutants were significantly decreased by TTX treatment (Figure 6E,F; SI Figure 5). Furthermore, nuclear levels of all the constructs decreased following TTX treatment (SI Figure 6A,B). Thus, activity-dependent untethering of ADAR2 from Pin1 is not a primary mechanism responsible for the loss of ADAR2 during TTX mediated upscaling.

\section{$\underline{\text { TTX mediated scaling enhances proteasomal degradation of ADAR2 }}$}

It has been proposed that ADAR2 is ubiquitinated and degraded in the cytosol (32). We therefore tested the effects of TTX on ADAR2 stability in the presence or absence of the proteasomal inhibitor Bortezomib (BTZ) (33). Consistent with suppression of synaptic activity leading to enhanced proteasomal degradation, BTZ prevented TTX-evoked decreases in ADAR2 (Figure 7A,B) and resulted in the accumulation of ubiquitinated products (Figure 7A,C). We next performed nuclear and cytoplasmic fractionations to determine if ADAR2 is exported from the nucleus for degradation in the cytosol. BTZ prevented the TTX-evoked decrease in ADAR2 in both the nuclear and cytosolic fractions, and actually led to a significant accumulation of ADAR2 in the cytosol (Figure 7D,E,F). Thus, ADAR2 may be exported to the cytosol to be ubiquitinated or ubiquitinated in the nucleus and exported to the cytosol to be degraded. In either case TTX induces proteasomal degradation of ADAR2.

\section{Blocking ADAR2 degradation prevents KAR up-scaling in response to TTX}

Since BTZ prevents the loss of ADAR2 in the nucleus in response to TTX, we next tested if preventing ADAR2 degradation with BTZ blocks TTX up-scaling of GluK2. Indeed, surface biotinylation showed that BTZ prevents TTX-induced GluK2 upscaling (Figure 8A,B) with no effect on EGFR (Figure 8A,C). These results support the hypothesis that ADAR2 degradation mediates KAR up-scaling. 


\section{Discussion}

\section{Selective ADAR2 mediated Q/R editing of GluK2 containing KARs regulates up-scaling}

Similar to AMPARs and NMDARs, GluK2 containing KARs up-scale following chronic suppression in synaptic activity (25). Here we show that the same TTX treatment leads to proteasomal degradation of ADAR2 and, consequently, reduced Q/R editing of GluK2. These data are consistent with activity-dependent loss of ADAR2 leading to the alteration in KAR editing which, in turn, directly mediates KAR up-scaling via increased KAR assembly and ER exit of unedited GluK2(Q) compared to edited GluK2(R) $(9,25)$.

Partial ADAR2 knockdown mimicked the decrease in ADAR2 levels observed after TTX scaling and did not alter GluA2 editing. We therefore used this tool to further study the role of ADAR2 in GluK2 up-scaling. Partial loss of ADAR2 phenocopies and occludes TTX-mediated GluK2 editing and upscaling. Thus, loss of ADAR2 is sufficient to up-scale KARs in the absence of TTX, and strongly suggests that reduced KAR editing underpins KAR up-scaling (Figure 9 schematic).

\section{Differential sensitivity of GluK2 to changes in ADAR2 levels}

While we observed robust up-scaling of both KARs (GluK2) and AMPARs (GluA2) by TTX, the mechanisms are different because the Q/R editing status of GluA2 was not altered. In line with this, complete loss of ADAR2 decreased GluA2 editing by $\sim 30 \%$ and GluK2 editing by $>60 \%$, whereas partial ADAR2 loss decreased editing of GluK2 by $\sim 20 \%$ but had no discernible impact on GluA2 editing. Notably, it has been reported that ADAR2 regulation of GluA2 is specifically confined to CA1 hippocampal and does not occur in CA3 (34). GluA2 is preferentially and almost completely Q/R edited, even when only low levels of ADAR2 are available. GluK2 mRNA, on the other hand, is more sensitive to changes in the levels of ADAR2. We interpret these data to indicate that the Q/R editing of KARs is more dynamically and activity-dependently regulated than AMPARs, and reason that this provides a rapidly tunable system to control KAR forward trafficking and scaling.

\section{Activity dependent regulation of ADAR2 proteostasis during TTX treatment}

ADAR2 acts in the nucleus where it is stabilised by Pin1. Loss of the ADAR2-Pin1 interaction leads to ADAR2 export into cytosol where it is ubiquitinated and degraded (32). Pin1 mediated stabilisation is important for ADAR2 editing activity during development in cortical neurons (11). However, our experiments suggest Pin1-ADAR2 interactions do not play a role in TTX up-scaling since both phosphonull and phosphomimetic mutants of ADAR2, which decrease or enhance binding to Pin1 respectively, were equally susceptible to the TTX mediated loss. 
TTX induces ADAR2 degradation which is blocked by the proteasome inhibitor BTZ. We speculate that ADAR2 accumulated in the cytosol can be re-imported into nucleus to maintain a functional pool of nuclear ADAR2. Consistent with this, blocking proteasomal degradation of ADAR2 prevents TTX induced GluK2 up-scaling. Thus, the loss of ADAR2 and consequent reduction in GluK2 Q/R editing are necessary and sufficient for KARs up-scaling.

KARs play key roles in neuronal excitability, synaptic transmission and plasticity, and network activity $(3,35)$. Moreover, KAR dysregulation is implicated in neuronal pathologies $(36,37)$. How KAR upscaling impacts on these pathways is an outstanding question. Our data provide novel mechanistic insight into how neuronal activity controls the surface expression and properties of KARs during homeostatic scaling that will have wide implication for neuronal function, and dysfunction in disease.

\section{Materials and Methods}

Primary neuronal cultures: Primary rat hippocampal neurones were dissected from E18 Wistar rat pups as previously described (38). Briefly neurones were dissected from E18 Wistar rats followed by trypsin dissociation and cultured for up to 2 weeks. For the first $24 \mathrm{~h}$, cells were grown in plating media: Neurobasal media (Gibco) supplemented with 10\% horse serum (Sigma), B27 (1x, Gibco), $\mathrm{P} / \mathrm{S}$ (100 units penicillin and $0.1 \mathrm{mg} / \mathrm{ml}$ streptomycin; ThermoScientific) and 5mM Glutamax (Gibco). After $24 \mathrm{~h}$, plating media was replaced with feeding media (Neurobasal containing 2mM Glutamax and lacking horse serum and P/S). For biochemistry experiments, cells were plated at a density of 500,000 per $35 \mathrm{~mm}$ well and 250,000 per coverslip for imaging experiments.

\section{ADAR2 cloning}

ADAR2 was cloned from rat neuronal cDNA and ADAR2 shRNA knockdown and knockdownrescue viruses were generated as previously described (38). ADAR2 was cloned from rat neuronal cDNA into the $\mathrm{Kpnl}$ and $\mathrm{Xbal}$ sites of the vector pcDNA3 with a HA tag at its $\mathrm{N}$-terminus.

Phosphomutants of ADAR2 were generated by site-directed mutagenesis. Pin1 was cloned from rat neuronal cDNA into the EcoRI and BamHI sites of the vector pEGFP-N1.

\section{$\underline{\text { Lentivirus generation }}$}

For ADAR2 knockdown experiments, shRNA sequences targeting ADAR2 cloned into a modified pXLG3-GFP vector (38) under the control of a $\mathrm{H} 1$ promoter. The ADAR2 target sequences were:

Complete shRNA AAGAACGCCCTGATGCAGCTG

Partial shRNA AACAAGAAGCTTGCCAAGGCC 
bioRxiv preprint doi: https://doi.org/10.1101/308650; this version posted April 25, 2018. The copyright holder for this preprint (which was not certified by peer review) is the author/funder, who has granted bioRxiv a license to display the preprint in perpetuity. It is made available under aCC-BY-NC-ND 4.0 International license.

For rescue experiments, shRNA-insensitive HA-ADAR2 was cloned into a modified pXLG3-GFP vector under the control of an SFFV promoter.

The viruses were produced in HEK293T cells as reported previously (38), harvested and added to DIV 9/10 hippocampal neurons for 5 days and lysed accordingly. For $24 \mathrm{~h}$ treatment experiments, cells were treated with on the $4^{\text {th }}$ day after virus addition and harvested accordingly on the $5^{\text {th }}$ day after the completion of the time course.

\section{Scaling, developmental and TTX timecourse and BTZ treatment}

For scaling experiments, cells were treated with $1 \mu \mathrm{M}$ TTX (Tocris) for $24 \mathrm{~h}$ and were either lysed directly in $1 \mathrm{x}$ sample buffer and heated for 10 minutes at $95^{\circ} \mathrm{C}$ or were used for either surface biotinylation or fractionation experiments (see below). For the TTX timecourse, cells were harvested directly into $1 \mathrm{x}$ sample buffer (4 $\mathrm{x}$ sample buffer $(0.24 \mathrm{M}$ Tris-HCL, $8 \%$ SDS, $40 \%$ glycerol, $10 \%$-mercaptoethanol and $0.009 \%$ Bromophenol blue) diluted in water). In experiments inhibiting proteosomal degradation, cells were treated with bortezomib (BTZ; Cell Signalling) dissolved in DMSO for $20 \mathrm{~h}$ at $1 \mu \mathrm{M}$ concentration. The control cells were treated with an equal volume of DMSO.

\section{Cell surface biotinylation and streptavidin pulldown}

Cell surface biotinylation was performed essentially as previously described (25). All steps were performed on ice with ice-cold buffers unless stated otherwise. Live hippocampal neurones post stated treatments were washed twice in phosphate buffered saline (PBS). Surface proteins were labelled with membrane impermeable Sulfo-NHS-SS biotin $(0.3 \mathrm{mg} / \mathrm{ml}$, Thermo Scientific) for 10 mins on ice and washed $3 x$ with PBS. $100 \mathrm{mM} \mathrm{NH}_{4} \mathrm{Cl}$ was added to quench free biotin-reactive groups and cells were extracted with lysis buffer $(50 \mathrm{mM}$ Tris $\mathrm{pH} 7.4,150 \mathrm{mM} \mathrm{NaCl}, 1 \%$ triton, $0.1 \%$ SDS, protease inhibitors (Roche)), incubated on ice for 30 mins and centrifuged $\left(15,000 \mathrm{~g}, 4^{\circ} \mathrm{C}, 20\right.$ mins) to remove cell debris. For streptavidin pulldown, each lysate was added to $30 \mu \mathrm{l}$ of streptavidin beads (Sigma) and left on a wheel to rotate for 90 minutes at $4^{\circ} \mathrm{C}$. The beads were then washed $3 x$ with wash buffer (lysis buffer without protease inhibitors) and proteins eluted with $2 x$ sample buffer and boiled for 10 minutes at $95^{\circ} \mathrm{C}$. The samples were then resolved by SDSPAGE and immunoblotted.

\section{Subcellular fractionation}

All the steps were performed on ice with ice-cold buffers unless stated otherwise. Following stated treatments, the cells were washed with PBS followed by addition of buffer 1 (150mM NaCl, $50 \mathrm{mM}$ HEPES $\mathrm{pH} 7.4,25 \mu \mathrm{g} / \mathrm{ml}$ Digitonin and protease inhibitors), incubated for 20 minutes, scraped, homogenised and centrifuged at $15000 \mathrm{~g}$ for 30 minutes at $4^{\circ} \mathrm{C}$. The supernatant consisted of the cytosolic proteins, while the pellet was resuspended in buffer 2 (Buffer 1 with $1 \%$ triton), incubated for 20 minutes and again centrifuged at $15000 \mathrm{~g}$ for 30 minutes at $4^{\circ} \mathrm{C}$. The supernatant consisted 
of mitochondrial proteins while the pellet was resuspended in buffer $3(150 \mathrm{mM} \mathrm{NaCl}, 50 \mathrm{mM}$ HEPES pH 7.4, $0.5 \%$ sodium deoxycholate, $0.1 \%$ SDS, protease inhibitors and $0.5 \%$ triton), incubated for $1 \mathrm{~h}$ and centrifuged at $15000 \mathrm{~g}$ for 30 minutes at $4^{\circ} \mathrm{C}$. The supernatant was discarded and the pellet, consisting of nuclear proteins, was resuspended in buffer 3 . The cytosolic supernatant was concentrated using 4 volumes of acetone (kept at $-20^{\circ} \mathrm{C}$ ), incubated at $-20^{\circ} \mathrm{C}$ for 1 $\mathrm{h}$ and spun for 20 minutes at $1500 \mathrm{~g}$ and resuspended in buffer 3 . BCA assay was then performed to determine protein concentrations and allow equal loading.

\section{BCA Assay}

BCA Assay was performed using a commercial kit (Pierce, Thermo Scientific). Following 30 minutes incubation at $37^{\circ} \mathrm{C}$, the samples were read using a plate reader (Versamax Microplate reader, Molecular devices) at a wavelength of $562 \mathrm{~nm}$.

\section{Western blotting and antibodies}

Antibodies used: GluK2 (1:1000, Millipore, rabbit polyclonal), GluA2 (1:1000, Synaptic Systems, rabbit polyclonal), ADAR2 (1:1000, Sigma, rabbit polyclonal), GAPDH (1:10,000, Abcam, mouse monoclonal), RhoGDI (1:1000, Abcam, rabbit polyclonal), Lamin B (1:1000, Santa Cruz, Goat polyclonal), EGFR (1:1000, Abcam, rabbit polyclonal), HA (1:2000, mouse, Sigma), Pin1 (mouse monoclonal, 1:1000, Santa cruz), ADAR1 (mouse monoclonal, 1;1000, Santa Cruz) and GFP (rat monoclonal, 1:10,000, Chromotek). Western blots were imaged and quantified using LI-COR Image Studio software or developed on X-ray film in a dark room using developer and fixer solutions. The blots were then scanned and quantified using FIJI ImageJ studio. Surface levels were normalised to their respective total levels. Nuclear protein levels were normalised to LaminB and cytosolic protein levels to RhoGDI. Treated samples were normalised to their control samples.

\section{RNA extraction, RT-PCR, and Bbvl digestion and RT-qPCR}

RNA samples were extracted from DIV14/15 hippocampal neurons following the stated treatments using RNeasy Mini Kit (Qiagen) according to the manufacturer's instructions. $1 \mu \mathrm{g}$ of RNA was used per condition and reverse transcribed to cDNA using RevertAid First Strand cDNA Synthesis Kit (Thermo Scientific) following the manufacturer's instructions. The following primers (spanning the M2 region of GluK2(39) and GluA2) were used, giving a PCR product of 452bp and 252bp:

\section{GluK2 F: 5'-GGTATAACCCACACCCTTGCAACC-3' \\ GluK2 R: 5'-TGACTCCATTAAGAAAGCATAATCCGA-3' \\ GluA2 F: 5'-GTGTTTGCCTACATTGGGGTC-3' \\ GluA2 R: 5'-TCCTCCTACACGGCTAACTTA-3'}

$5 \mu \mathrm{l}$ of cDNA was used to set up PCR reactions [50 $\mu$ l total, 35 cycles, 20 s denaturing at $95^{\circ} \mathrm{C}, 10 \mathrm{~s}$ annealing at $60^{\circ} \mathrm{C}$ and $15 \mathrm{~s}$ elongation at $70^{\circ} \mathrm{C}$. 
To determine the level of RNA editing, Bbvl (New England Biolabs) digestion was used as previously (39). Total $20 \mu$ l digestion was set up using $10 \mu \mathrm{l}$ of PCR product at $37^{\circ} \mathrm{C}$ for $2 \mathrm{~h}$. All of the digested product was run on $4 \%$ agarose gel and the ethidium bromide stained bands were imaged using UV transilluminator and quantified using FIJI NIH ImageJ. To determine the level of editing in GluK2, the following formula was used: [Intensity of 376 (edited)/Intensity of (376 (edited) + 269 (unedited))] ${ }^{\star} 100$. The band at $76 \mathrm{bp}$ allowed to determine equal loading. For GluA2, [Intensity of 158 (edited)/Intensity of (158 (edited) +94 (unedited)) $]^{\star} 100$.

Purified PCR products were also sent for sequencing to Eurofins Genomics at $4 \mathrm{ng} / \mu \mathrm{l}$ along with the above GluK2 and GluA2 F primers, to obtain sequence chromatographs.

For RT-qPCR, $2 \mu$ of the cDNA samples per condition were mixed with PowerUp SYBR green Master Mix (Life Technologies) and forward and reverse primers targeting ADAR2 and GAPDH and amplified quantitatively using Real Time PCR System (MiniOpticon, BioRAD) for 40 cycles and $\mathrm{Ct}$ values were recorded. Each reaction was performed in triplicate and average $\mathrm{Ct}$ was measured per condition. ADAR2 $\mathrm{Ct}$ values were normalised to GAPDH Ct values and ADAR2 mRNA fold difference value of TTX treated conditions was normalised against the untreated control. Melting curve of the primers were also determined to ensure the specificity of the primers and lack of primer dimer formation. The primers used were: ADAR2 F - TCCCGCCTGTGTAAGCAC, GAPDH AATCCCATCACCATCTTCCA.

\section{$\underline{\text { GFP trap }}$}

GFP-trap protocols were as previously published (40). HEK293T cells were transfected the next day using Lipofectamine ${ }^{\mathrm{TM}} 3000$ and $2.5 \mu \mathrm{g}$ of each construct. $48 \mathrm{~h}$ post-transfection, cells were washed with PBS and lysed and harvested in lysis buffer (20mM Tris pH7.4, $137 \mathrm{mM} \mathrm{NaCl}, 2 \mathrm{mM}$ sodium pyrophosphate, 2mM EDTA, 1\% triton X-100, 0.1\% SDS, 25mM $\beta$-glycerophosphate, 10\% glycerol, protease inhibitors (Roche), phosphatase inhibitor cocktail 2 (1:100, Sigma)). The lysates were left to incubate for 30 minutes on ice and centrifuged at $1500 \mathrm{~g}$ for 20 minutes at $4^{\circ} \mathrm{C}$ to remove any cell debris. The supernatant was then added to $5 \mu$ l of GFP-trap beads (Chromotek), incubated on wheel at $4^{\circ} \mathrm{C}$ for 90 minutes and washed $3 \mathrm{x}$ with wash buffer (lysis buffer without protease or phosphatase inhibitors). The samples were then lysed in $2 x$ sample buffer, heated at $95^{\circ} \mathrm{C}$ for 10 minutes and separated using SDS-PAGE.

\section{Fixed immunostaining, imaging and analysis}

Immunostaining was performed as previously described (41). For fixed immunostaining, cells post TTX treatment or lentiviral treatment as indicated were fixed with $4 \%$ formaldehyde for 10 minutes, washed 3 times with PBS, treated with $100 \mathrm{mM}$ glycine to quench any remaining formaldehyde and washed 3 times with PBS. The cells were permeabilised and blocked with 3\% BSA in PBS and $0.1 \%$ triton for 30 minutes. The cells were then incubated for $1 \mathrm{~h}$ with primary antibodies (anti-ADAR2 
(Abcam, rabbit) 1:400, anti-Fibrillarin (Abcam, mouse) 1:400 and anti-HA (Sigma, mouse) 1:600) in $3 \%$ BSA at room temperature, washed 3 times for 5 minutes each with PBS and incubated for 45 minutes with the indicated secondary antibodies (Jackson Immunoresearch Antibodies, 1:400) in $3 \%$ BSA at room temperature. Three $\times 5$ minute washes were performed with PBS and the cells were mounted using DAPI containing fluoromount.

A Leica SP7 confocal microscope was used to image the coverslips. FIJI NIH Image $\mathrm{J}$ was used to compress the z-stacks and analyse the mean intensity per nucleus using the DAPI channel to draw regions of interest. To calculate the percentage of cells expressing ADAR2, all the cells expressing ADAR2 were manually counted per image taken.

\section{Statistical Analysis}

Mean value were calculated for all data and all error bars show standard deviation. All statistical analysis was performed using GraphPad Prism software version 7.0 as stated.

\section{Acknowledgements}

We are grateful to the MRC, BBSRC and Wellcome Trust for financial support. SG and AJE were supported by Wellcome Trust Dynamic Cell Biology PhD studentships. We thank Dr Yasuko Nakamura for excellent technical and logistical support.

\section{Author Contributions}

SG performed all of the experiments. AJE and KAW provided specialised constructs, advice and assisted in some experiments. JMH supervised the research. All authors contributed to writing the manuscript. 
bioRxiv preprint doi: https://doi.org/10.1101/308650; this version posted April 25, 2018. The copyright holder for this preprint (which was not certified by peer review) is the author/funder, who has granted bioRxiv a license to display the preprint in perpetuity. It is made available under aCC-BY-NC-ND 4.0 International license.

\section{References}

1. Petralia RS, Wang YX, \& Wenthold RJ (1994) Histological and ultrastructural localization of the kainate receptor subunits, KA2 and GluR6/7, in the rat nervous system using selective antipeptide antibodies. J Comp Neurol 349(1):85-110.

2. Kumar J, Schuck P, \& Mayer ML (2011) Structure and assembly mechanism for heteromeric kainate receptors. Neuron 71(2):319-331.

3. Evans AJ, Gurung S, Henley JM, Nakamura Y, \& Wilkinson KA (2017) Exciting Times: New Advances Towards Understanding the Regulation and Roles of Kainate Receptors. Neurochem Res.

4. Griffith TN \& Swanson GT (2015) Identification of critical functional determinants of kainate receptor modulation by auxiliary protein Neto2. J Physiol 593(22):4815-4833.

5. Egebjerg J, Kukekov V, \& Heinemann SF (1994) Intron sequence directs RNA editing of the glutamate receptor subunit GluR2 coding sequence. Proc Natl Acad Sci USA 91(22):1027010274.

6. Howe JR (1996) Homomeric and heteromeric ion channels formed from the kainate type subunits GluR6 and KA2 have very small, but different unitary conductances. J. Neurophysiol. 76:510-519.

7. Sommer B, Kohler M, Sprengel R, \& Seeburg PH (1991) RNA Editing in Brain Controls a Determinant of Ion Flow in Glutamate-Gated Channels. Cell 67(1):11-19.

8. Nishikura K (2016) A-to-I editing of coding and non-coding RNAs by ADARs. Nat Rev Mol Cell Biol 17(2):83-96.

9. Ball SM, Atlason PT, Shittu-Balogun OO, \& Molnar E (2010) Assembly and intracellular distribution of kainate receptors is determined by RNA editing and subunit composition. $J$ Neurochem 114(6):1805-1818.

10. Swanson GT, Feldmeyer D, Kaneda M, \& Cull-Candy SG (1996) Effect of RNA editing and subunit co-assembly single-channel properties of recombinant kainate receptors. J Physiol 492(Pt 1):129-142.

11. Behm M, Wahlstedt $\mathrm{H}$, Widmark A, Eriksson M, \& Ohman M (2017) Accumulation of nuclear ADAR2 regulates adenosine-to-inosine RNA editing during neuronal development. $J$ Cell Sci 130(4):745-753.

12. Paschen W, Schmitt J, Gissel C, \& Dux E (1997) Developmental changes of RNA editing of glutamate receptor subunits GluR5 and GluR6: in vivo versus in vitro. Brain Res Dev Brain Res 98(2):271-280.

13. Bernard A, et al. (1999) Q/R editing of the rat GluR5 and GluR6 kainate receptors in vivo and in vitro: evidence for independent developmental, pathological and cellular regulation. Eur. J. Neurosci. 11:604-616.

14. Filippini A, Bonini D, La Via L, \& Barbon A (2016) The Good and the Bad of Glutamate Receptor RNA Editing. Mol Neurobiol.

15. Higuchi M, et al. (2000) Point mutation in an AMPA receptor gene rescues lethality in mice deficient in the RNA-editing enzyme ADAR2. Nature 406(6791):78-81.

16. Vissel B, et al. (2001) The role of RNA editing of kainate receptors in synaptic plasticity and seizures. Neuron 29(1):217-227.

17. Bliss TV, Collingridge GL, \& Morris RG (2014) Synaptic plasticity in health and disease: introduction and overview. Philos Trans R Soc Lond B Biol Sci 369(1633):20130129.

18. Malenka RC \& Bear MF (2004) LTP and LTD: an embarrassment of riches. Neuron 44(1):521. 
bioRxiv preprint doi: https://doi.org/10.1101/308650; this version posted April 25, 2018. The copyright holder for this preprint (which was not certified by peer review) is the author/funder, who has granted bioRxiv a license to display the preprint in perpetuity. It is made available under aCC-BY-NC-ND 4.0 International license.

19. Lu W, et al. (2001) Activation of synaptic NMDA receptors induces membrane insertion of new AMPA receptors and LTP in cultured hippocampal neurons. Neuron 29(1):243-254.

20. Petrovic MM, et al. (2017) Metabotropic action of postsynaptic kainate receptors triggers hippocampal long-term potentiation. Nat Neurosci 20(4):529-539.

21. Chamberlain SE, et al. (2012) SUMOylation and phosphorylation of GluK2 regulate kainate receptor trafficking and synaptic plasticity. Nat Neurosci 15(6):845-852.

22. Martin S \& Henley JM (2004) Activity-dependent endocytic sorting of kainate receptors to recycling or degradation pathways. EMBO J 23(24):4749-4759.

23. Martin S, Bouschet T, Jenkins EL, Nishimune A, \& Henley JM (2008) Bidirectional regulation of kainate receptor surface expression in hippocampal neurons. J Biol Chem 283(52):3643536440.

24. González-González IM, et al. (2012) Kainate Receptor Trafficking. WIRES Membrane Trasnsport and Signalling 1(1):31-44.

25. Evans AJ, Gurung S, Wilkinson KA, Stephens DJ, \& Henley JM (2017) Assembly, Secretory Pathway Trafficking, and Surface Delivery of Kainate Receptors Is Regulated by Neuronal Activity. Cell Rep 19(12):2613-2626.

26. Turrigiano G (2011) Too many cooks? Intrinsic and synaptic homeostatic mechanisms in cortical circuit refinement. Annu Rev Neurosci 34:89-103.

27. Mu Y, Otsuka T, Horton AC, Scott DB, \& Ehlers MD (2003) Activity-dependent mRNA splicing controls ER export and synaptic delivery of NMDA receptors. Neuron 40(3):581-594.

28. Turrigiano GG, Leslie KR, Desai NS, Rutherford LC, \& Nelson SB (1998) Activity-dependent scaling of quantal amplitude in neocortical neurons. Nature 391(6670):892-896.

29. Turrigiano GG (2008) The self-tuning neuron: synaptic scaling of excitatory synapses. Cell 135(3):422-435.

30. Faundez V, Krauss R, Holuigue L, Garrido J, \& Gonzalez A (1992) Epidermal growth factor receptor in synaptic fractions of the rat central nervous system. J Biol Chem 267(28):2036320370.

31. Herb A, Higuchi M, Sprengel R, \& Seeburg PH (1996) Q/R site editing in kainate receptor GluR5 and GluR6 pre-mRNAs requires distant intronic sequences. Proc Natl Acad Sci U S A 93(5):1875-1880.

32. Marcucci R, et al. (2011) Pin1 and WWP2 regulate GluR2 Q/R site RNA editing by ADAR2 with opposing effects. EMBO J 30(20):4211-4222.

33. Chen D, Frezza M, Schmitt S, Kanwar J, \& Dou QP (2011) Bortezomib as the first proteasome inhibitor anticancer drug: current status and future perspectives. Curr Cancer Drug Targets 11(3):239-253.

34. Balik A, Penn AC, Nemoda Z, \& Greger IH (2013) Activity-regulated RNA editing in select neuronal subfields in hippocampus. Nucleic Acids Res 41(2):1124-1134.

35. Contractor A, Mulle C, \& Swanson GT (2011) Kainate receptors coming of age: milestones of two decades of research. Trends Neurosci 34(3):154-163.

36. Lerma J \& Marques JM (2013) Kainate receptors in health and disease. Neuron 80(2):292311.

37. Crepel V \& Mulle C (2015) Physiopathology of kainate receptors in epilepsy. Curr Opin Pharmacol 20:83-88.

38. Rocca DL, Wilkinson KA, \& Henley JM (2017) SUMOylation of FOXP1 regulates transcriptional repression via CtBP1 to drive dendritic morphogenesis. Sci Rep 7(1):877. 
bioRxiv preprint doi: https://doi org/10 1101/308650; this version posted April 25 2018. The copyright holder for this preprint (which was not certified by peer review) is the author/funder, who has granted bioRxiv a license to display the preprint in perpetuity. It is made available under aCC-BY-NC-ND 4.0 International license.

39. Bernard A, et al. (1999) Q/R editing of the rat GluR5 and GluR6 kainate receptors in vivo and in vitro: evidence for independent developmental, pathological and cellular regulation. The European journal of neuroscience 11(2):604-616.

40. Guo C, Wilkinson KA, Evans AJ, Rubin PP, \& Henley JM (2017) SENP3-mediated deSUMOylation of Drp1 facilitates interaction with Mff to promote cell death. Sci Rep 7:43811.

41. Glebov OO, Tigaret CM, Mellor JR, \& Henley JM (2015) Clathrin-independent trafficking of AMPA receptors. J Neurosci 35(12):4830-4836. 
Figure 1. Chronic suppression of network activity with TTX increases surface levels of GluK2-containing KARs.

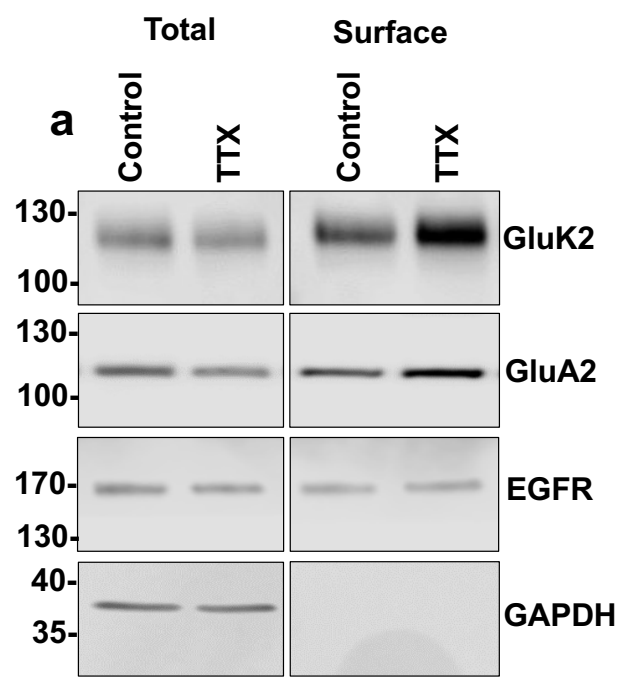

b

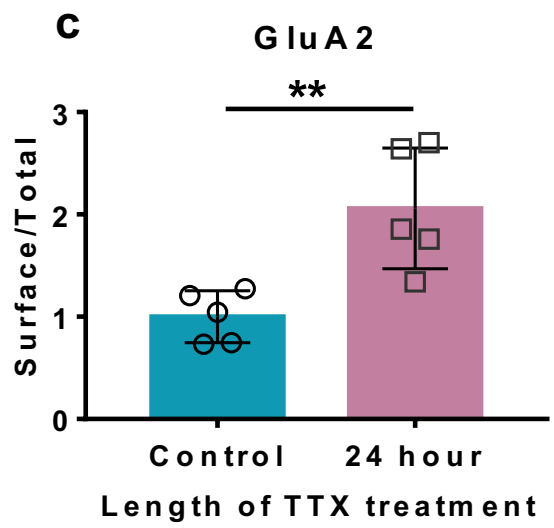

d
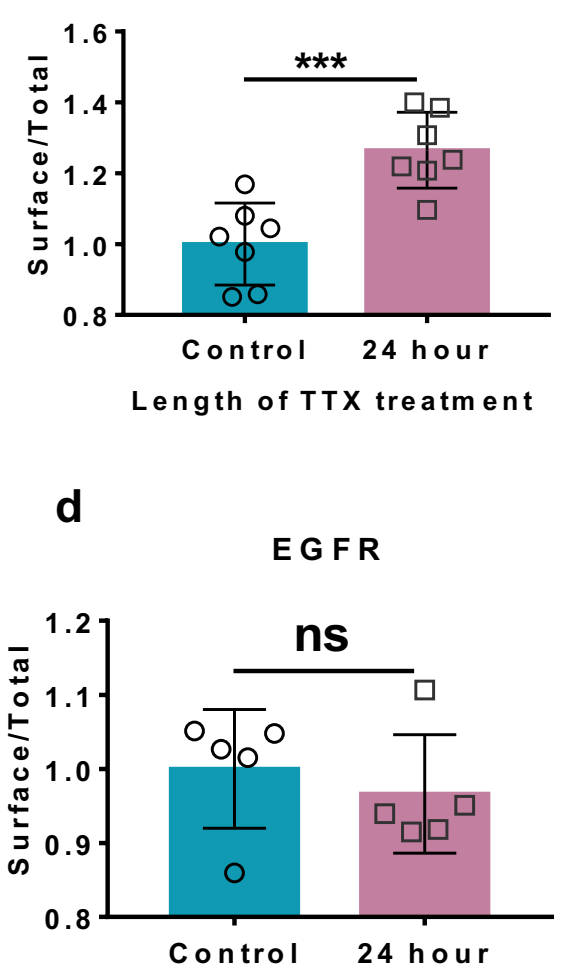

Length of TTX treatment

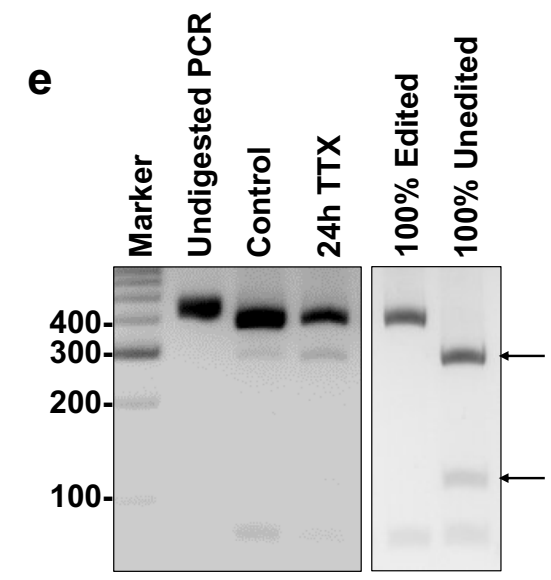

f

G luK2 Editing levels

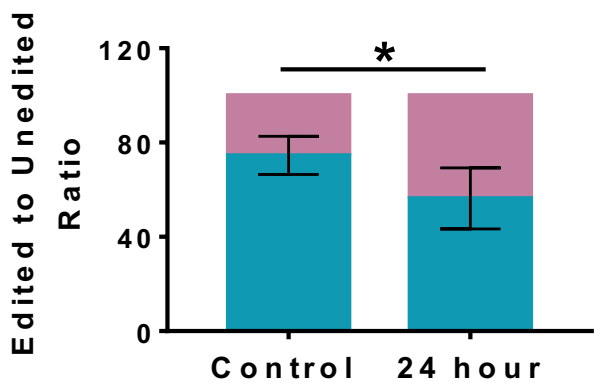

Length of TTX treatment 
bioRxiv preprint doi: https://doi.org/10.1101/308650; this version posted April 25, 2018. The copyright holder for this preprint (which was not certified by peer review) is the author/funder, who has granted bioRxiv a license to display the preprint in perpetuity. It is made available under aCC-BY-NC-ND 4.0 International license.

Figure 2. Chronic suppression of network activity with TTX decreases ADAR2 levels.
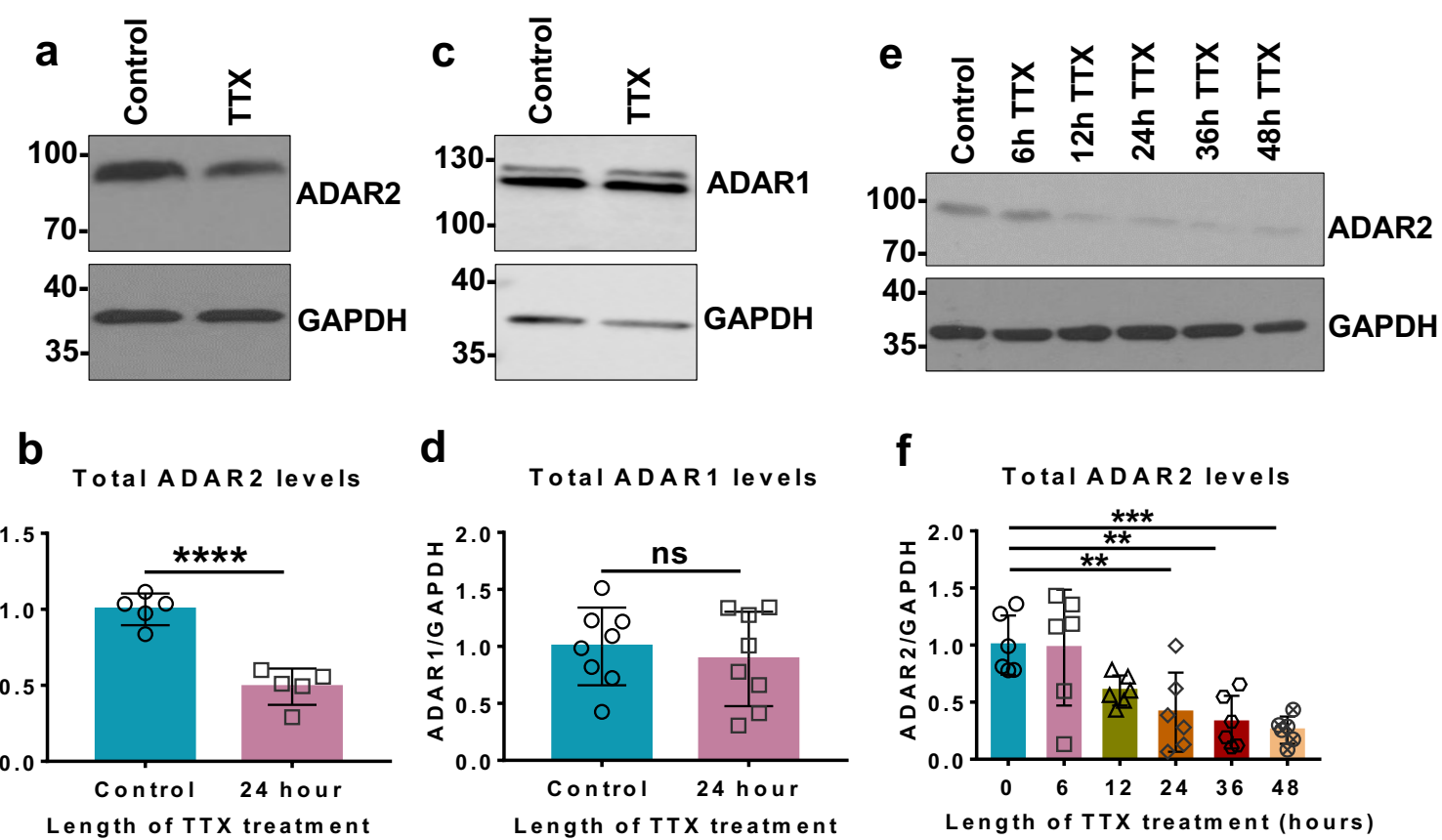

d

Total ADAR1 levels

\section{f}
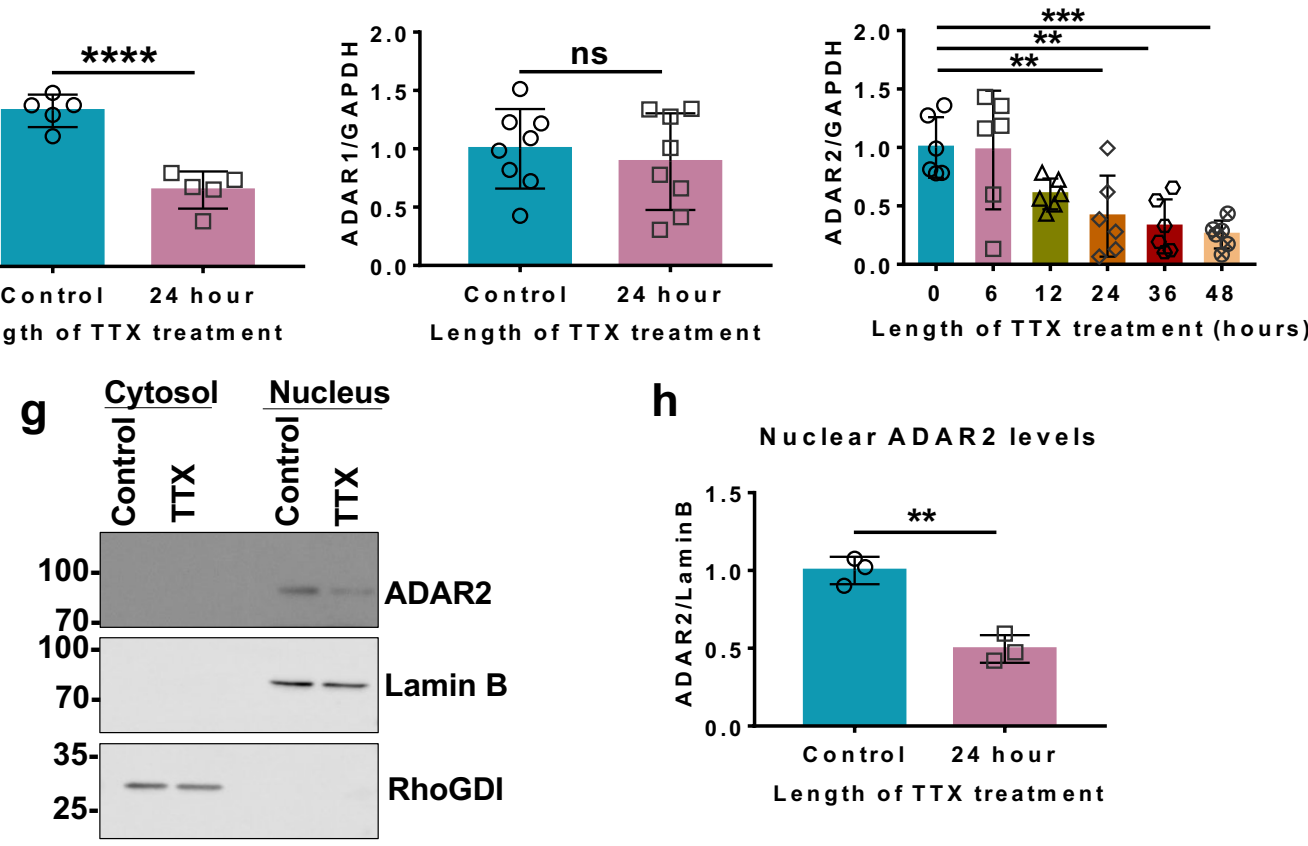

h

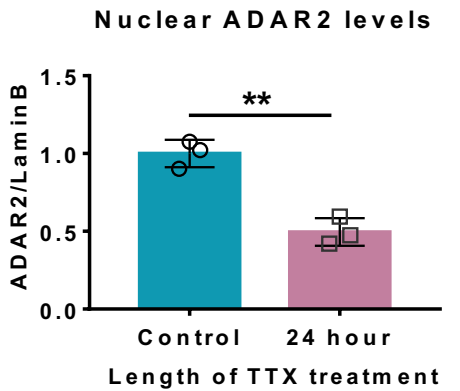

i

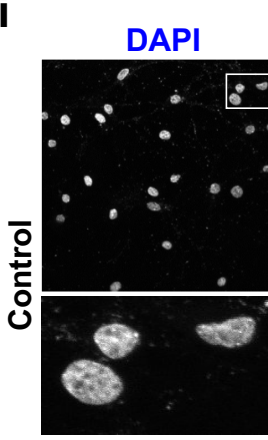

ADAR2
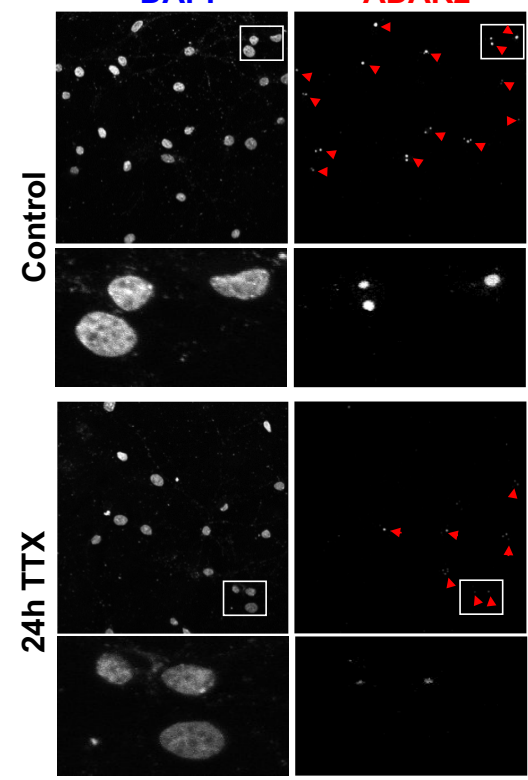
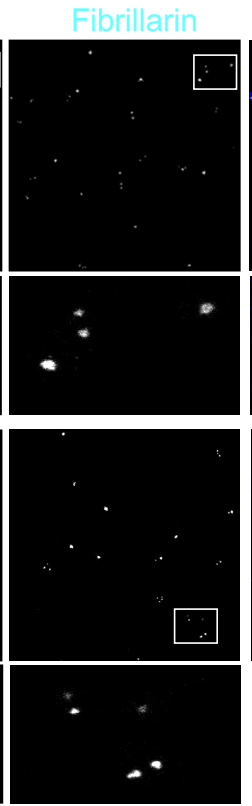

Overlay
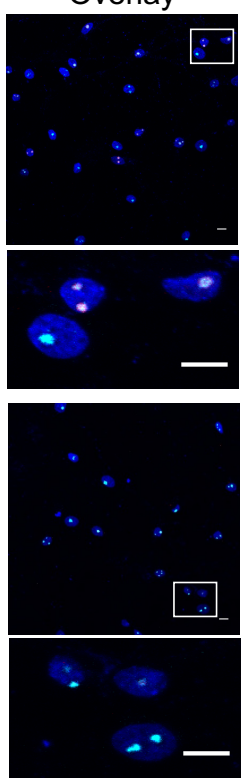

j

Nuclear ADAR 2 levels

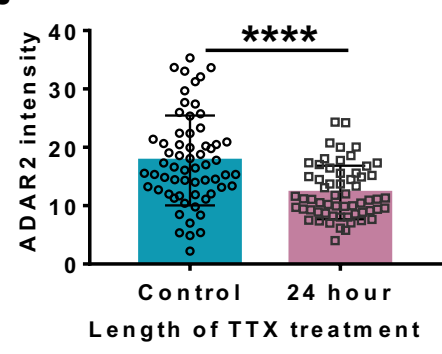

K Percentage of cells

expressing ADAR 2

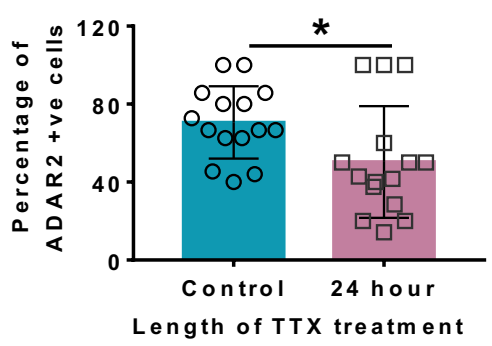


Figure 3. Complete and Partial ADAR2 knockdown

a

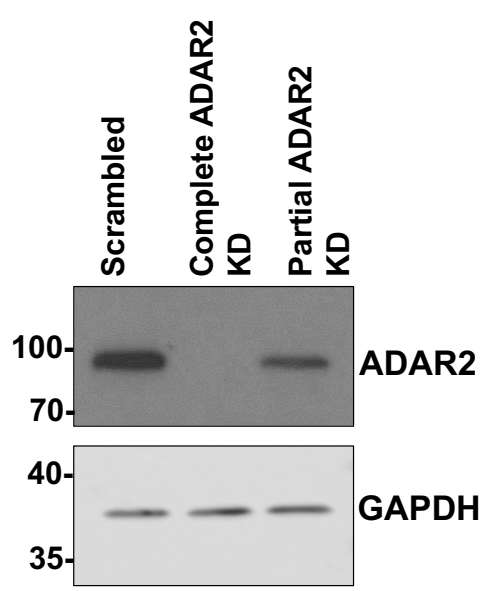

\section{C}

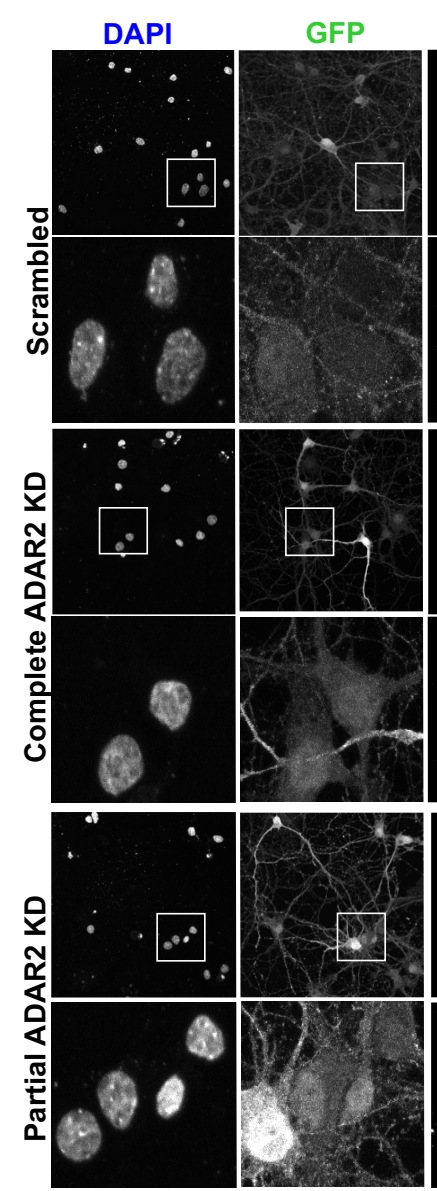

ADAR2
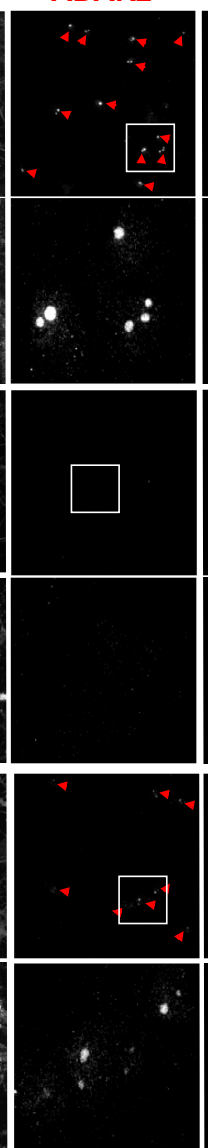

b

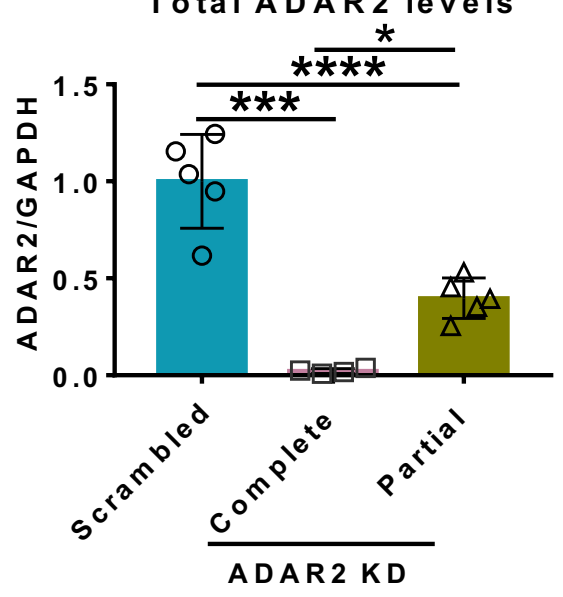

d

Overlay
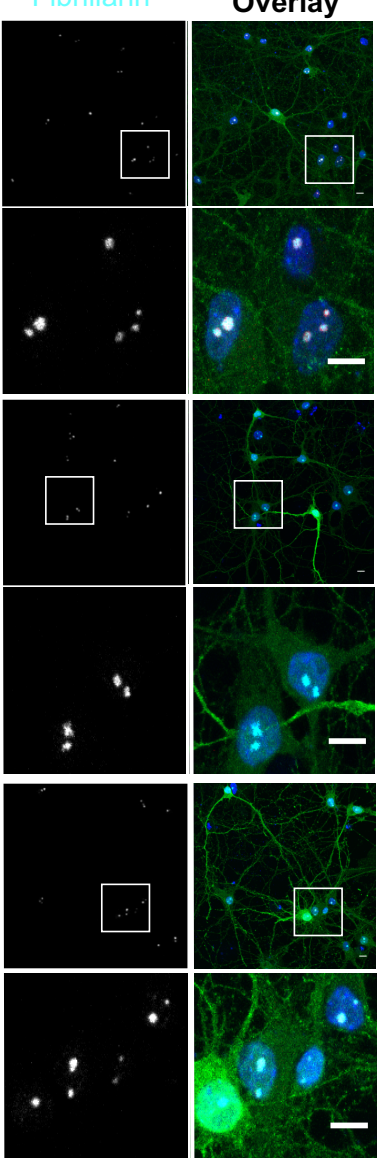

Nuclear ADAR2 levels

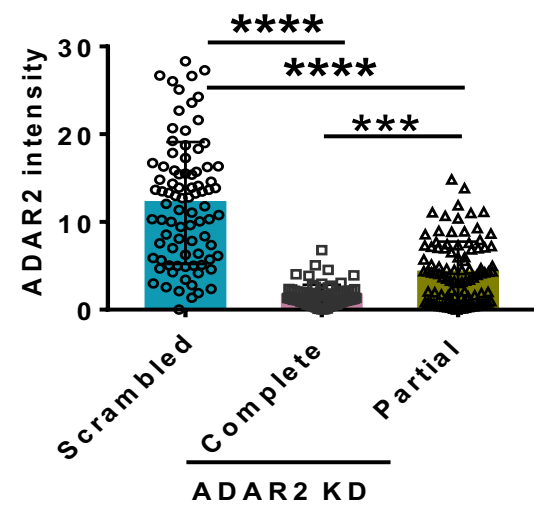

e Percentage of cells

expressing ADAR2

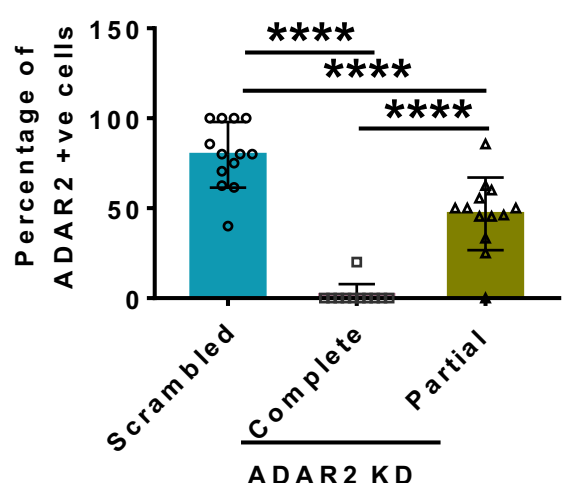


Figure 4. Complete and Partial ADAR2 knock down have differential effects on GluK2 Q/R editing

a

C

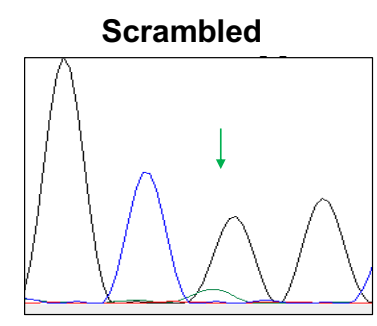

b

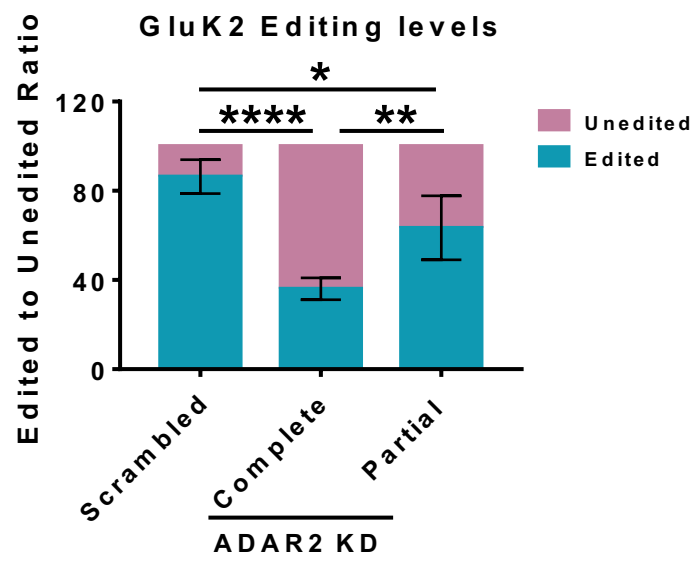

d

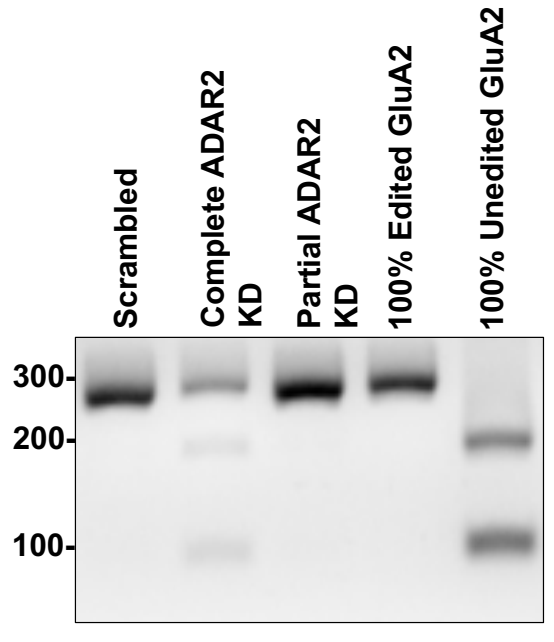

e

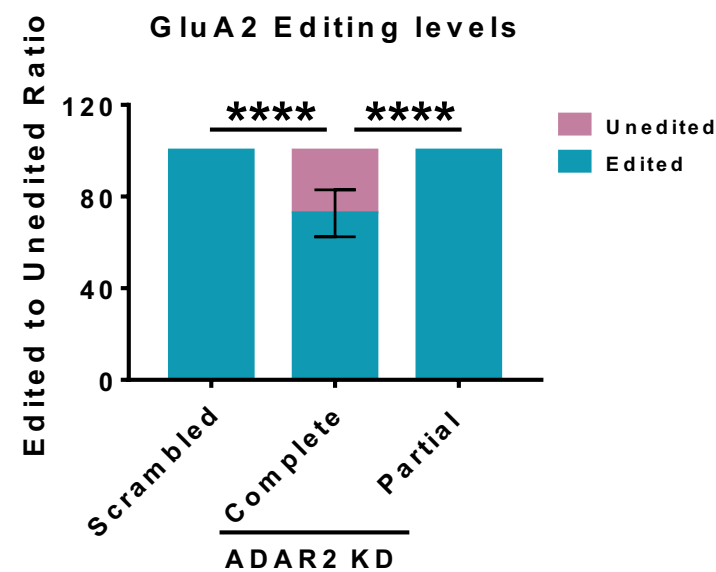

$\mathbf{f}$

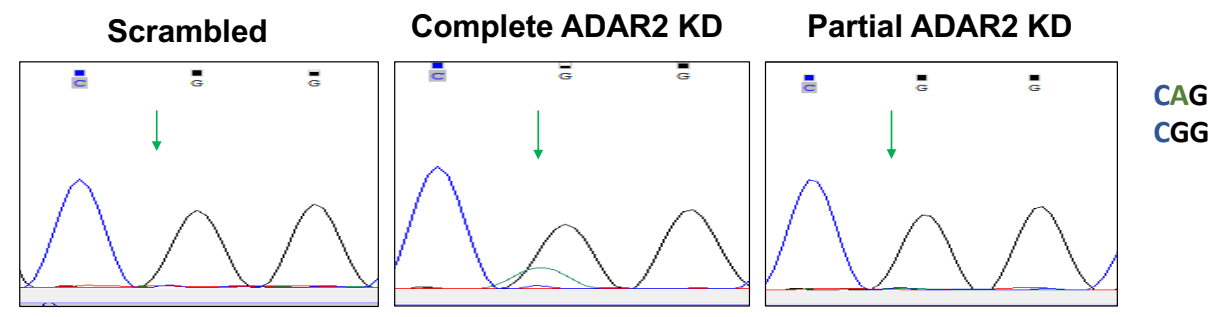


Figure 5. Partial ADAR2 knockdown phenocopies and occludes TTX upscaling of GluK2 containing KARs

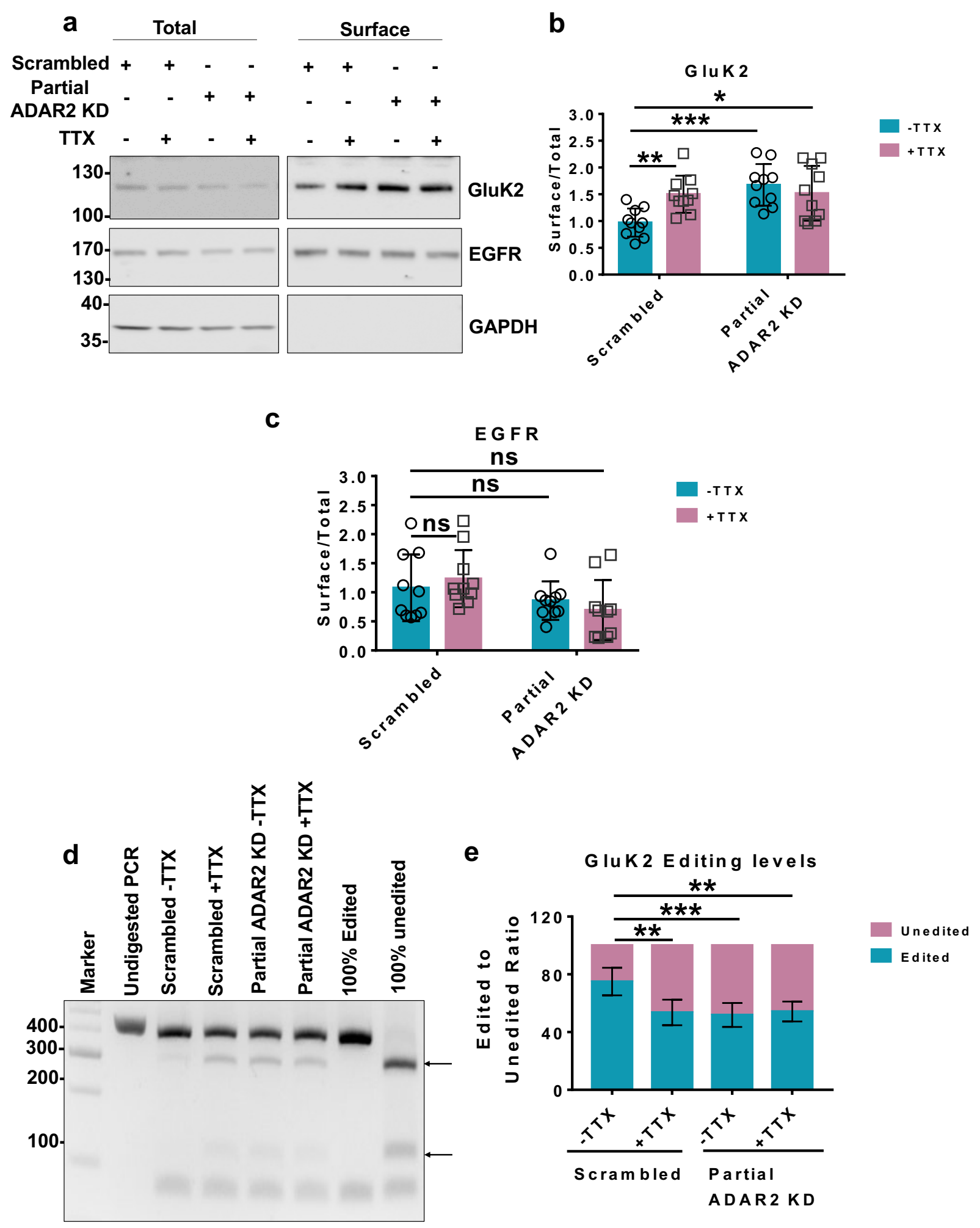


Figure 6. Loss of ADAR2 during scaling is not dependent on Pin1 or ADAR2 phosphorylation
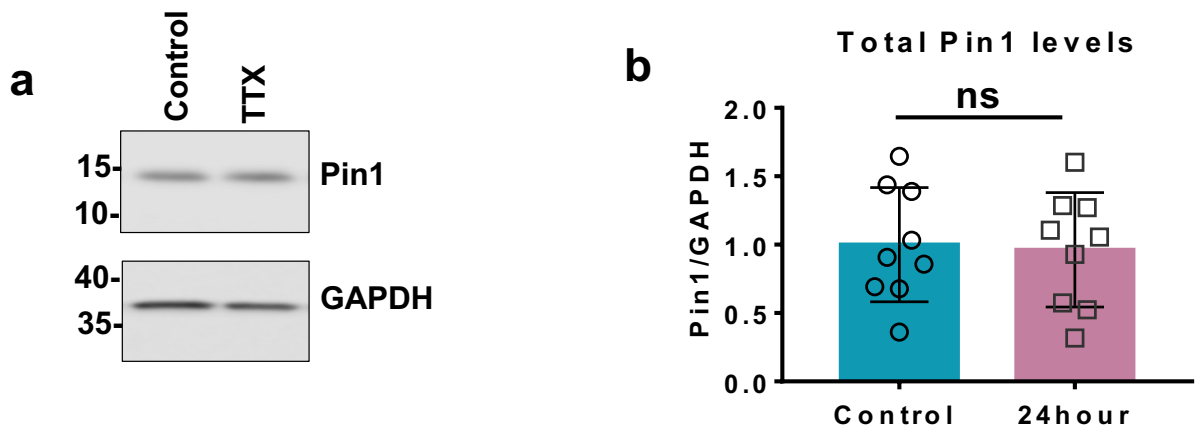

Length of TTX treatment

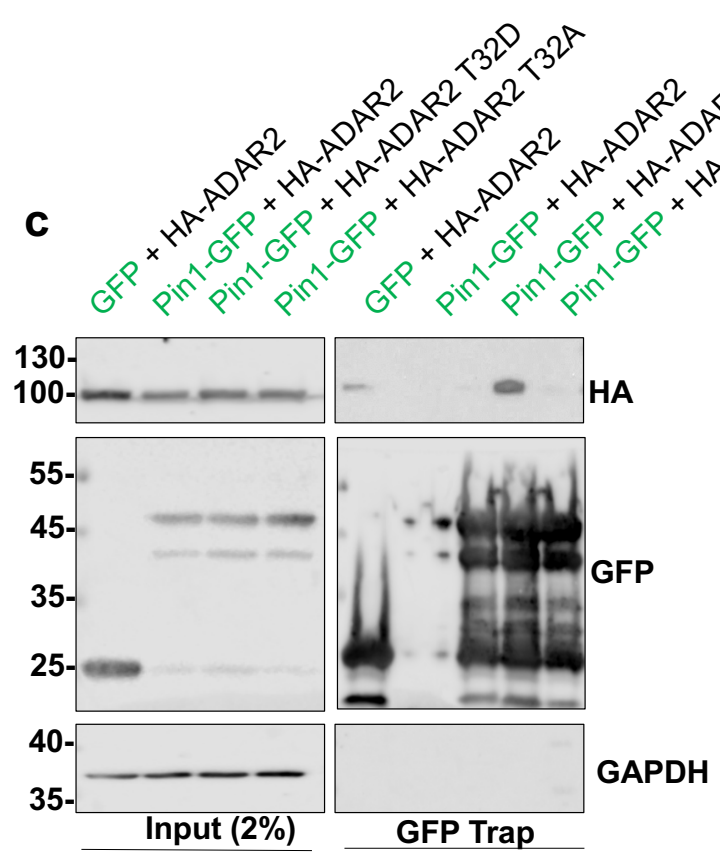

d
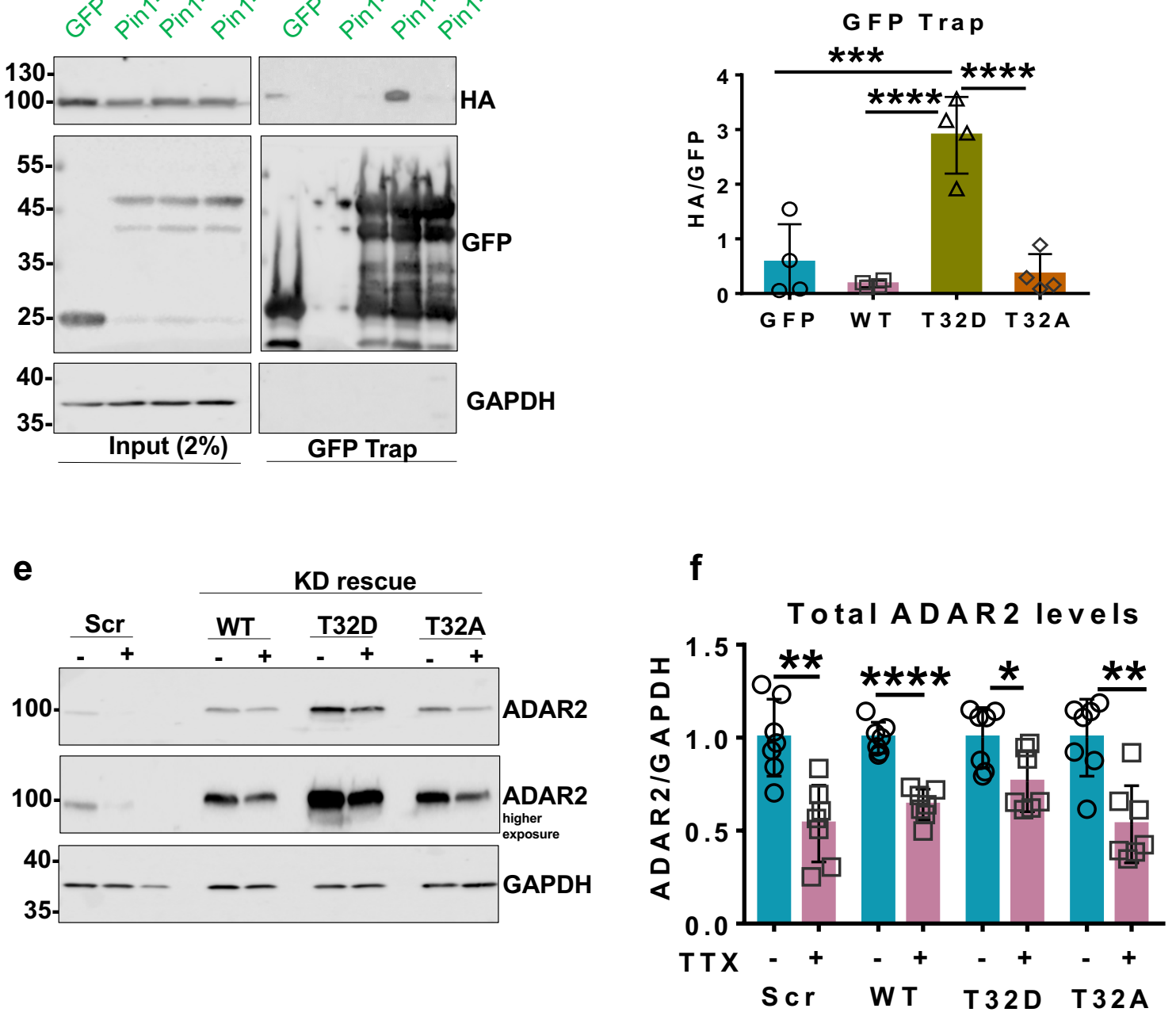
Figure 7. TTX mediated scaling enhances proteasomal degradation of ADAR2.

a

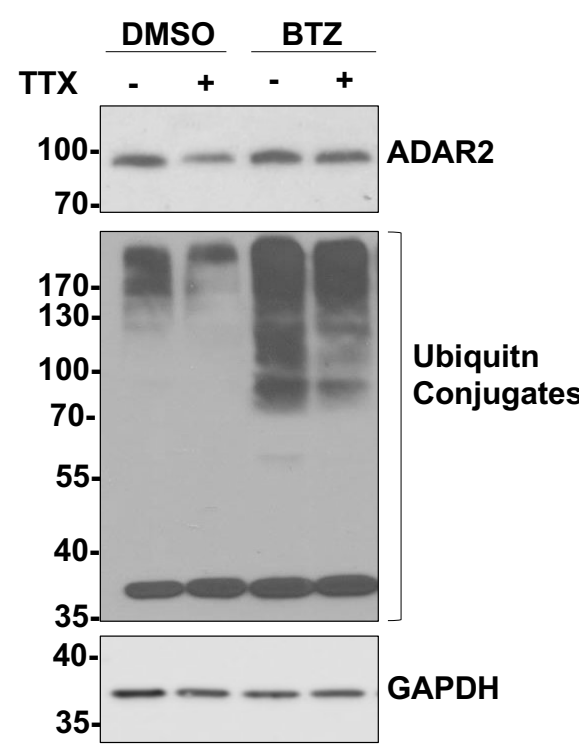

b

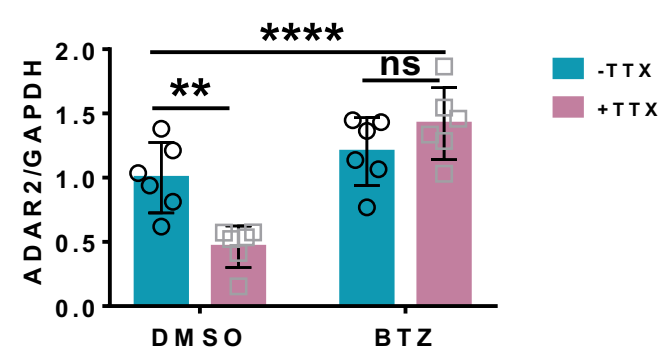

C

Total Ubiquitin Conjugates

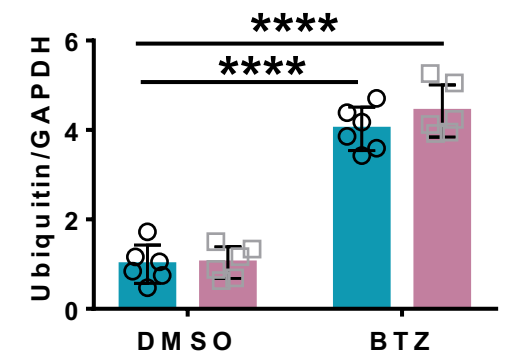

Nuclear ADAR 2 levels

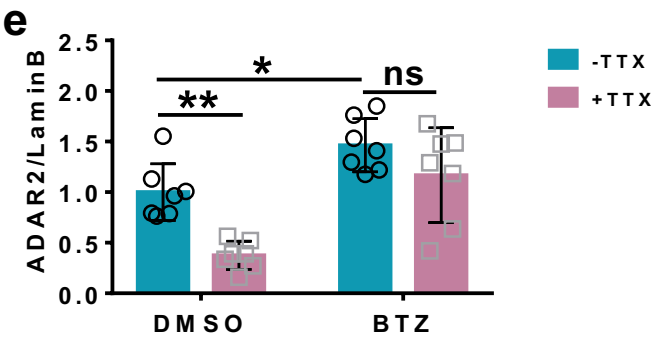

ADAR2

Higher

exposure

Lamin B

70.

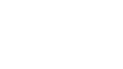

f

Cytosol ADAR2 levels

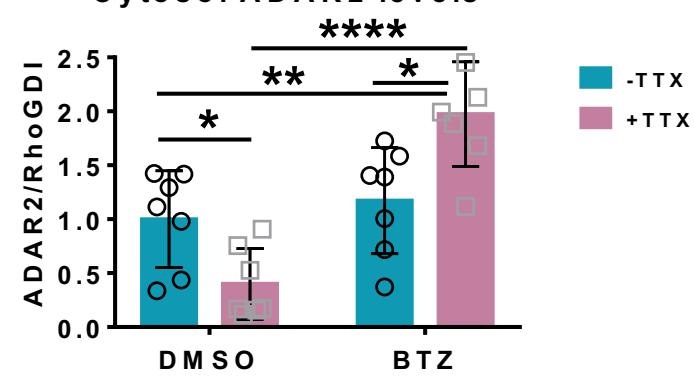


Figure 8. Blocking ADAR2 degradation with BTZ prevents KAR up-scaling in response to TTX

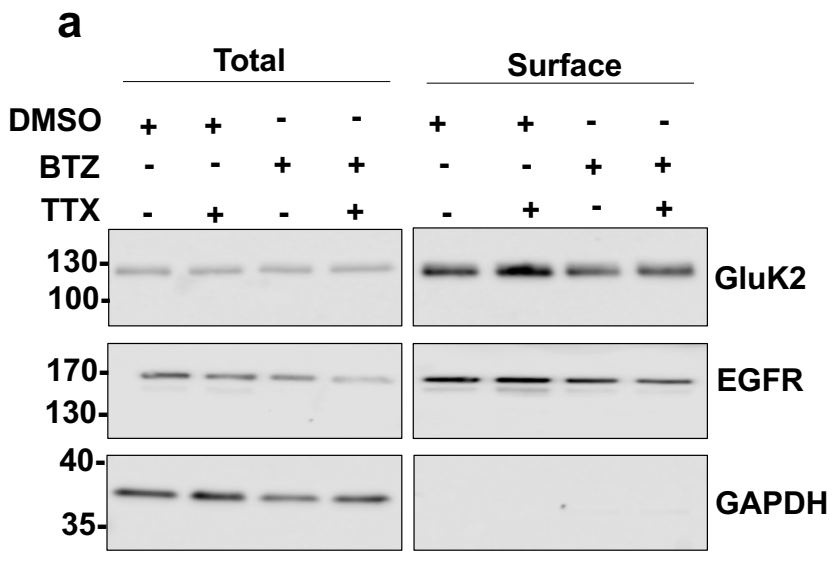

b

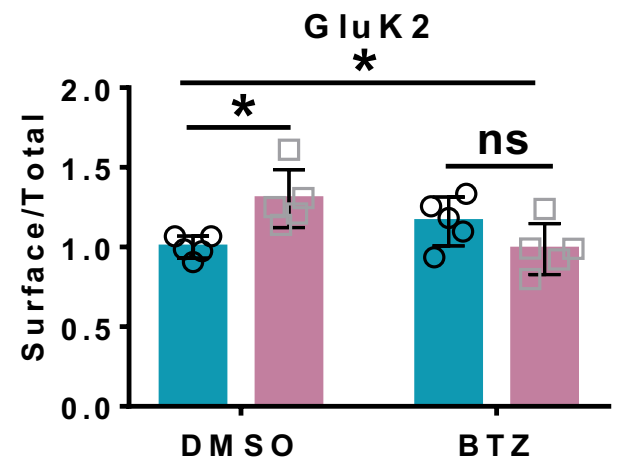

C

\section{E G F R}

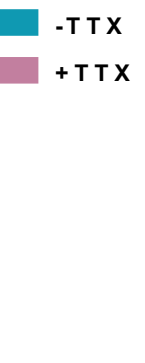

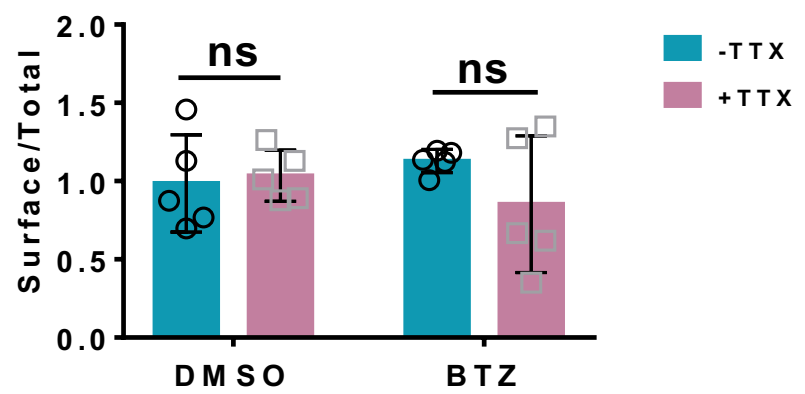


Figure 9. Schematic of ADAR2 mediated Q/R editing regulating GluK2 containing KARs homeostatic up-scaling.

\section{Suppression of}

\section{synaptic activity}

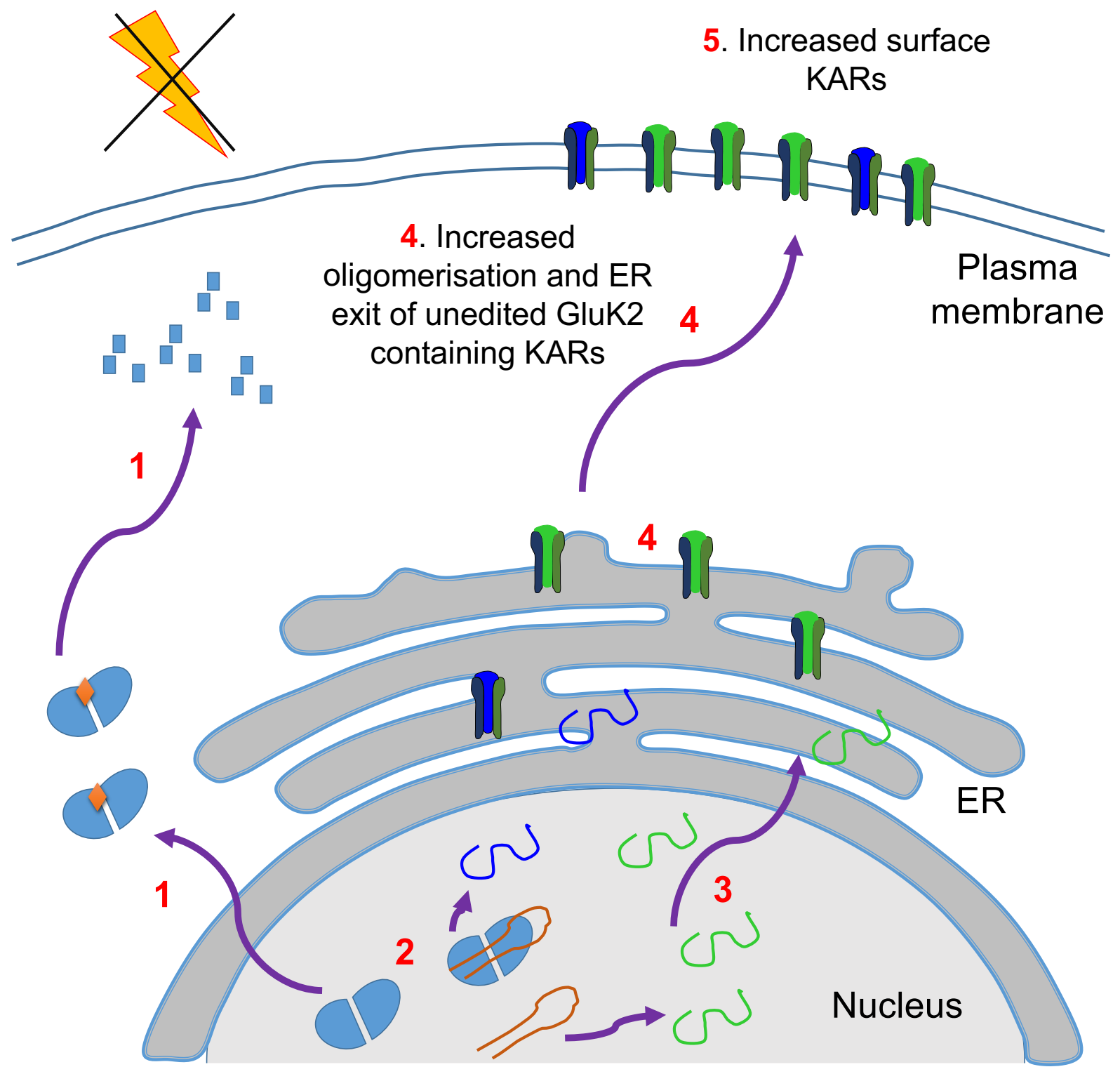

1. Nuclear export, ubiquitination and proteasomal degradation of ADAR2
2. Reduced ADAR2 levels and GluK2 editing
3. Increase in mature unedited GluK2 transcripts

ADAR2

Edited GluK2 KAR

Unedited GluK2

KAR

Ubiquitin

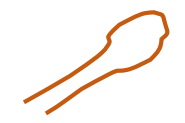
dsRNA

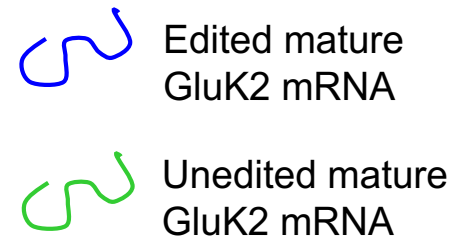




\section{Figure Legends:}

Figure 1: Chronic suppression of network activity with TTX increases surface levels of GluK2 containing KARs.

a. Representative western blot of total and surface levels of GluK2 (KAR subunit), GluA2 (AMPAR subunit), EGFR and GAPDH in hippocampal neurones with or without $24 \mathrm{~h}$ TTX treatment to suppress synaptic activity. EGFR was used as negative control and GAPDH was used as a control to show only surface proteins were labelled with biotin.

b. Quantification of surface levels of GluK2 (A) from 5 independent experiments. Surface levels were normalised to their total levels. Statistical Analysis; Unpaired t-test ${ }^{* * *}<0.001$.

c. Quantification of surface levels of GluA2 (A) from 5 independent experiments. Surface levels were normalised to their total levels. Statistical Analysis; Unpaired t-test ${ }^{* *}<0.01$.

d. Quantification of surface levels of EGFR (A) from 5 independent experiments. Surface levels were normalised to their total levels. Statistical Analysis; Unpaired t-test, ns $>0.05$.

e. RT-PCR and Bbvl digestion analysis of GluK2 Q/R editing from hippocampal neurones treated with or without TTX.

f. Quantification of E from 5 independent experiments. Statistical Analysis: Unpaired t-test; $*<0.05$.

Figure 2: Chronic suppression of network activity with TTX decreases ADAR2 levels.

a. Representative western blots of total ADAR2 and GAPDH levels in hippocampal neurons with or without $24 \mathrm{~h}$ TTX treatment to suppress synaptic activity.

b. Quantification of (A) total ADAR2 normalised to GAPDH from 5 independent experiments. ADAR2 levels normalised to loading control GAPDH. Statistical Analysis: Unpaired T test; $* * * *<0.0001$.

c. Representative western blots of total ADAR1 level and GAPDH levels in hippocampal neurones with or without $24 \mathrm{~h}$ TTX treatment.

d. Quantification of $(C)$ total ADAR1 normalised to GAPDH from 8 independent experiments. Both bands were quantified. Statistical Analysis: Unpaired T test; ns>0.05.

e. Representative western blots showing total ADAR2 and GAPDH levels with increasing lengths of TTX treatment.

f. Quantification of (E) total ADAR2 normalised to GAPDH from 6 independent experiments. Statistical Analysis: One Way ANOVA Dunnett's multiple comparison test; ${ }^{* *}<0.01$, $* * *<0.001$. 
g. Representative western blot of nuclear ADAR2 levels in hippocampal neurones with or without $24 \mathrm{~h}$ TTX treatment. Cell fractionation was performed to determine the ADAR2 levels in the nucleus. Lamin B was used as a nuclear marker and RhoGDI as cytosol marker.

h. Quantification of $(G)$ nuclear ADAR2 immunoblots normalised to Lamin B from 3 independent experiments. Statistical Analysis: Unpaired T test; ${ }^{* *}<0.01$.

i. Representative images of hippocampal neurons with or without $24 \mathrm{~h}$ TTX treatment labelled with nuclear DAPI stain (blue), anti-ADAR2 (red) and anti-Fibrillarin (nucleolar marker; cyan). Bottom panels show zoom in images as indicated and the red arrows indicate cells expressing ADAR2.

j. Quantification of (I) ADAR2 intensity per nucleus. $N=3$ independent dissections and $n=60$ cells for control and 62 cells for TTX treated. Statistical Analysis: Wilcoxon matched-pairs signed rank test, ${ }^{* * *}<0.0001$.

k. Analysis of percentage of cells expressing ADAR2 (I) with or without TTX. N=3 independent dissections and $n=15$ fields of view. Statistical Analysis: Unpaired t-test, ${ }^{*}<0.05$.

Figure 3: Complete and partial ADAR2 knockdown.

a. Representative western blots of total ADAR2 and GAPDH levels in hippocampal neurons treated with either scrambled, complete or partial ADAR2 KDs.

b. Quantification of (A) total ADAR2 normalised to GAPDH from 5 independent experiments for scrambled and partial knockdown and 4 independent experiments for complete knockdown.. Statistical Analysis: One-Way ANOVA with Tukey's multiple comparisons test; ${ }^{*}<0.05$, $* * *<0.001,{ }^{* * * *}<0.0001$.

c. Representative images of hippocampal neurons imaged for DAPI (blue), GFP (green, lentivirus infected cells), ADAR2 (red) and Fibrillarin (nucleolar marker; cyan) for cells infected with either scrambled, complete or partial ADAR2 KD. Bottom panels show zoom in images as indicated and the red arrows indicate cells expressing ADAR2.

d. Quantification of (C) ADAR2 intensity per nucleus. $N=3$ independent dissections and $n=75$ cells (complete KD), 86 cells (scrambled) and 99 cells (Partial knockdown). Statistical Analysis: One Way Anova with Tukey's multiple comparisons test, ${ }^{* * *}<0.001,{ }^{* * * *}<0.0001$.

e. Quantification of (C) percentage of cells expressing ADAR2. N=3 independent dissections and $n=11-13$ fields of view. Statistical Analysis: One Way Anova with Tukey's multiple comparisons test, ${ }^{* * *}<0.0001$.

Figure 4: Complete and partial ADAR2 knockdown differentially alter GluK2 and GluA2 Q/R editing. 
a. RT-PCR and Bbvl digestion analysis of GluK2 Q/R editing from hippocampal neurons infected with either scrambled, complete or partial ADAR2 KDs.

b. Quantification of $(A)$ from 4 independent experiments. Statistical Analysis: One Way ANOVA with Tukey's multiple comparisons test; ${ }^{*}<0.05,{ }^{* *}<0.01,{ }^{* * * *}<0.0001$.

c. Sanger sequencing chromatographs of the GluK2 PCR products from hippocampal neurons infected with either scrambled, complete or partial ADAR2 KDs, showing dual A and G peaks at the editing site indicated by the green arrows. The green peak represents an $A$ base read and black represents a $\mathrm{G}$ base read.

d. RT-PCR and Bbvl digestion analysis of GluA2 Q/R editing from hippocampal neurons infected with either scrambled, complete or partial ADAR2 KDs.

e. Quantification of (D) from 4 independent experiments. Statistical Analysis: One Way ANOVA with Tukey's multiple comparisons test; ${ }^{* * * *}<0.0001$.

f. Sanger sequencing chromatographs of the GluA2 PCR products from hippocampal neurons infected with either scrambled, complete or partial ADAR2 KDs. Green arrows indicate the editing site. Only samples treated with complete ADAR2 knockdown show a dual A and G peak at the editing site. The green peak represents an $A$ base read and black represents a $\mathrm{G}$ base read.

Figure 5: Partial ADAR2 knockdown phenocopies and occludes TTX up-scaling of GluK2 containing KARs.

a. Representative western blot of total and surface levels of GluK2, EGFR and GAPDH in scrambled or ADAR2 KD infected cells in the presence or absence of TTX. EGFR was used as a negative control while GAPDH was used as a control to show only surface proteins were labelled with biotin.

b. Quantification of (A) surface levels of GluK2 from 10 independent experiments. Surface levels were normalised to their total levels. Statistical Analysis: Two-Way ANOVA with Tukey's Multiple comparisons test; ${ }^{*}<0.05,{ }^{* *}<0.01,{ }^{* * *}<0.001$.

c. Quantification of (A) surface levels of EGFR from 10 independent experiments. Surface levels were normalised to their total levels. Statistical Analysis: Two-Way ANOVA with Tukey's Multiple comparisons test; ns>0.05.

d. RT-PCR and Bbvl digestion analysis of GluK2 Q/R editing from hippocampal neurons infected with either scrambled or partial ADAR2 KD with or without TTX.

e. Quantification of (D) from 5 independent experiments. Statistical Analysis: One Way ANOVA with Tukey's multiple comparisons test; ${ }^{* *}<0.01,{ }^{* * *} 0.001$. 
Figure 6: Loss of ADAR2 during TTX mediated scaling is not dependent on Pin1 or ADAR2 phosphorylation

a. Representative western blots of total Pin1 and GAPDH levels in neurons with or without TTX treatment for $24 \mathrm{~h}$.

b. Quantification of (A) Pin1 levels normalised to GAPDH from 9 independent experiments. Statistical Analysis; Unpaired t-test: ns>0.05.

c. Representative blots of GFP-trap performed in HEK293T cells where Pin-GFP was overexpressed with either HA-WT ADAR2, HA-T32D ADAR2 or HA-T32A ADAR2. The pulldowns were blotted for HA, GFP and GAPDH. Free GFP was overexpressed with WTADAR2 as a negative control.

d. Quantification of $(C)$ showing interaction of HA-T32D ADAR2 with Pin1-GFP. The HA signal was normalised to the GFP. N=4 independent experiments. Statistical Analysis: One-way ANOVA with Tukey's multiple comparisons test; ${ }^{* * *}<0.001,{ }^{* * * *}<0.0001$.

e. Representative western blots of total ADAR2 and GAPDH in cells infected with either scrambled or KD rescue lentiviruses expressing either WT ADAR2, T32D ADAR2 or T32A ADAR2, in the presence or absence of TTX.

f. Quantification of (E) total ADAR2 levels normalised to GAPDH from 7 independent experiments. Each TTX treated condition was normalised to their respective non-treated control. Statistical Analysis: Unpaired t-test; ${ }^{*}<0.05,{ }^{* *}<0.01,{ }^{* * *}<0.00,{ }^{* * * *}<0.0001$.

Figure 7: TTX mediated scaling enhances proteasomal degradation of ADAR2.

a. Representative western blots of total ADAR2, total ubiquitin and GAPDH levels in neurons treated with either DMSO or $1 \mu \mathrm{M}$ Bortezomib (BTZ) for $20 \mathrm{~h}$ either in the presence or absence of $24 \mathrm{~h}$ TTX.

b. Quantification of (A) total ADAR2 immunoblots normalised to GAPDH from 6 independent experiments. Statistical Analysis: Two-way ANOVA with Tukey's multiple comparisons test: ${ }^{* *}<0.01,{ }^{* * * *}<0.0001, \mathrm{~ns}>0.05$.

c. Quantification of $(A)$ total ubiquitin conjugated products normalised to GAPDH from 6 independent experiments. Statistical Analysis: Two-way ANOVA with Tukey's multiple comparisons test: ${ }^{* * * *}<0.0001$.

d. Representative western blots of ADAR2 in the nucleus and cytoplasm in the presence of DMSO or BTZ with or without TTX treatment. Lamin B was used as a nuclear marker and RhoGDI as cytosol marker. 
e. Quantification of (D) nuclear ADAR2 from 7 independent experiments. Nuclear ADAR2 levels were normalised to Lamin B. Statistical Analysis: Two-way ANOVA Tukey's multiple comparisons test: ${ }^{*}<0.05$, ${ }^{* *}<0.01$, ns $>0.05$.

f. Quantification of (D) cytosolic ADAR2 from 7 independent experiments. Cytosolic ADAR2 was normalised to RhoGDI level. Statistical Analysis: Two-way ANOVA with Tukey's multiple comparisons test: ${ }^{*}<0.05,{ }^{* *}<0.01,{ }^{* * * *}<0.0001$.

Figure 8: Blocking ADAR2 degradation with BTZ prevents KAR up-scaling in response to TTX.

a. Representative western blots of total and surface levels of GluK2, EGFR and GAPDH in DMSO and BTZ $(20 \mathrm{~h}, 1 \mu \mathrm{M})$ treated cells in the presence or absence of $24 \mathrm{~h}$ TTX. EGFR was used as a negative control while GAPDH was used as a control to show only surface proteins were labelled with biotin.

b. Quantification of (A) surface levels of GluK2 from 5 independent experiments. Surface levels were normalised to their total levels. Statistical Analysis: Two-Way ANOVA with Tukey's Multiple comparisons test; *<0.05, ns $>0.05$.

c. Quantification of (A) surface levels of EGFR from 5 independent experiments. Surface levels were normalised to their total levels. Statistical Analysis: Two-Way ANOVA with Tukey's Multiple comparisons test; ns>0.05.

Figure 9: Schematic of ADAR2 mediated Q/R editing regulating GluK2 containing KARs homeostatic up-scaling.

Under basal conditions unedited GluK2 transcripts are edited at their Q/R site by ADAR2 enzyme resulting in $\sim 80 \%$ edited mature GluK 2 transcripts. The resultant edited and unedited GluK2 subunits oligomerise in the ER and traffic into the surface. Under conditions of synaptic activity suppression with TTX treatment, ADAR2 undergoes proteasomal degradation in the cytosol (1). This results in less ADAR2 editing of GluK2 pre-mRNA transcripts (2) and increased levels of unedited GluK2 mature transcripts (3). The subsequent increase in the proportion of unedited GluK2(Q) allows enhanced oligomerisation and ER exit (4) to increase surface expression of GluK2 containing KARs on the surface (5). 


\section{Supplementary Figures}

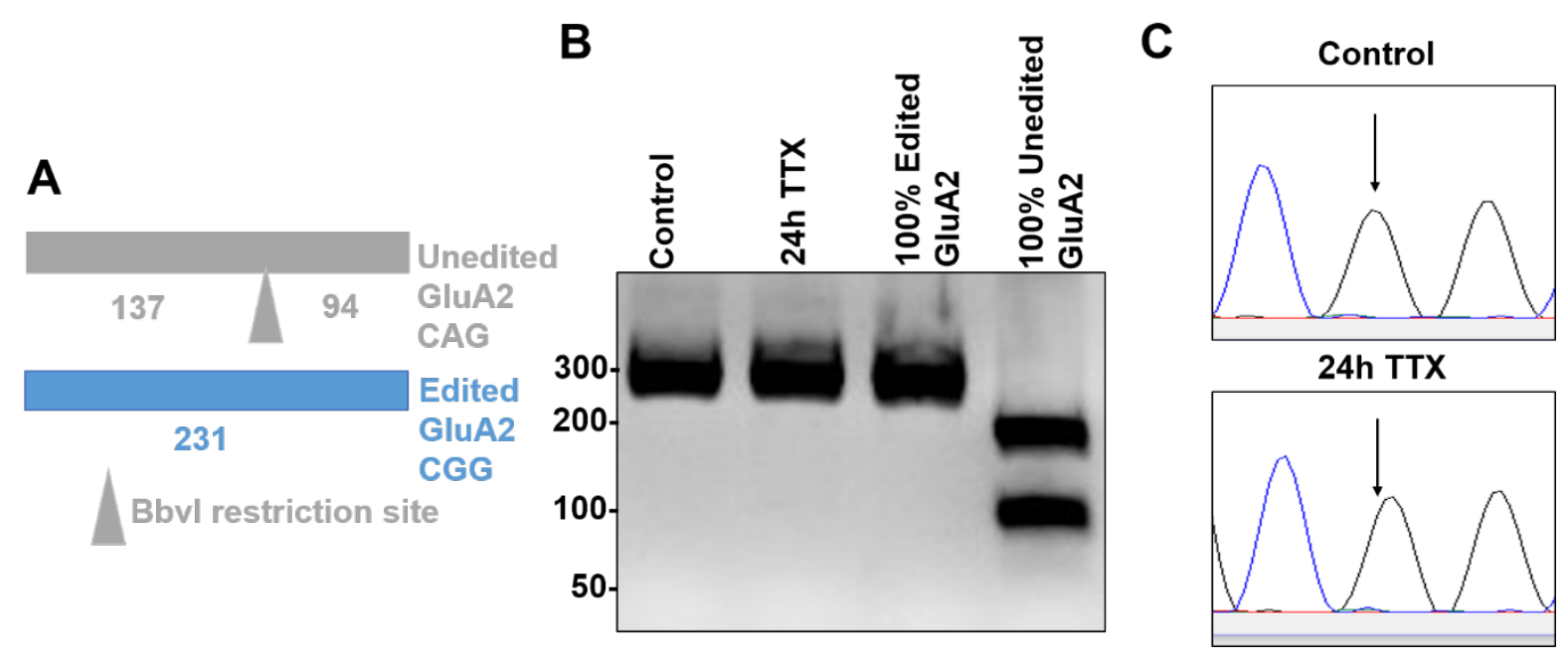

Supplementary Figure 1: GluA2 editing status does not change with TTX treatment despite the loss of ADAR2 levels.

A. Schematic of Bbvl digestion analysis on the PCR amplified M2 region of GluA2.

B. RT-PCR and Bbvl digestion analysis of GluA2 Q/R editing from hippocampal neurones treated with or without TTX.

C. Sanger sequencing chromatographs of the GluA2 PCR products from hippocampal neurones treated with or without TTX, showing no changes in the editing levels. Black arrows indicate the editing site. Green peak represents $A$ base read and black represents $\mathrm{G}$ base read. 
A

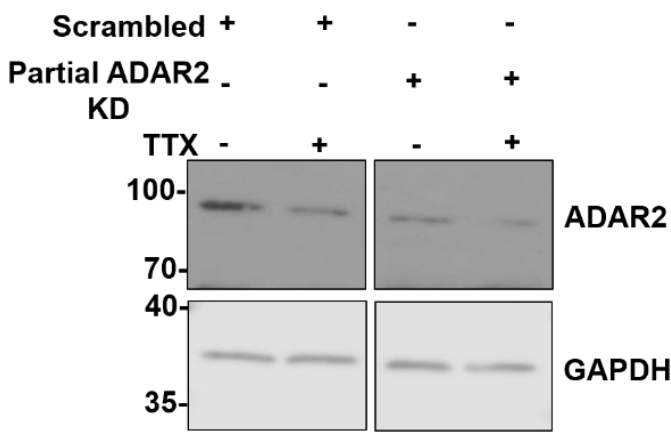

B

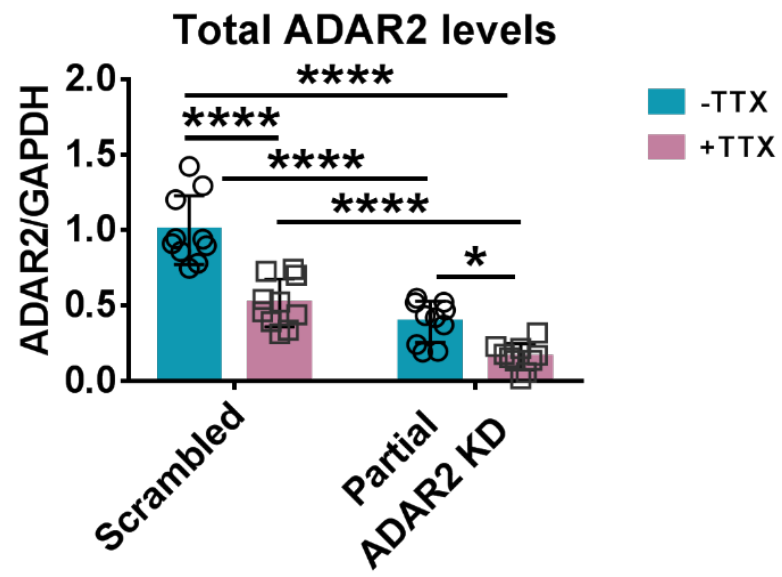

Supplementary Figure 2. ADAR2 level further decreases with TTX treatment in an already depleted system.

A. Representative western blots of total ADAR2 and GAPDH levels in cells infected with either scrambled or Partial ADAR2 KD either in the presence or absence of $24 \mathrm{~h}$ TTX.

B. Quantification of the (A) total ADAR2 immunoblots normalised to GAPDH from 10 independent experiments. Statistical Analysis: Two-way ANOVA with Tukey's multiple comparisons test: ${ }^{*}<0.05,{ }^{* * * *}<0.0001$. 


\section{A DAR 2 m R N A levels}

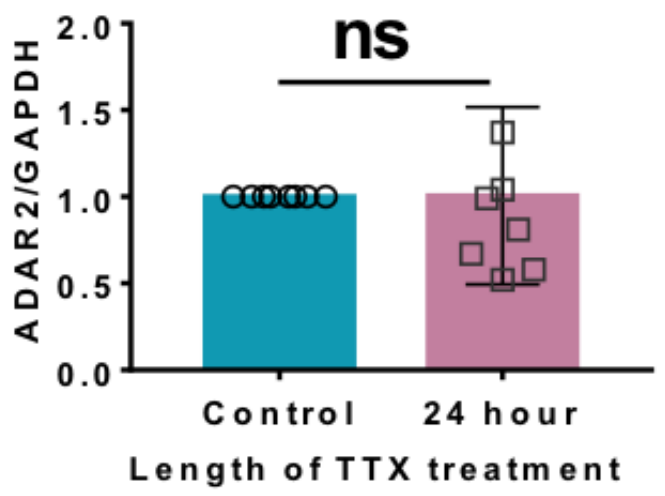

Supplementary Figure 3. RT-qPCR analysis of mRNA levels of ADAR2 post TTX treatment showing no changes in the ADAR2 mature mRNA transcripts.

Statistical analysis: Unpaired t-test; ns>0.05. 

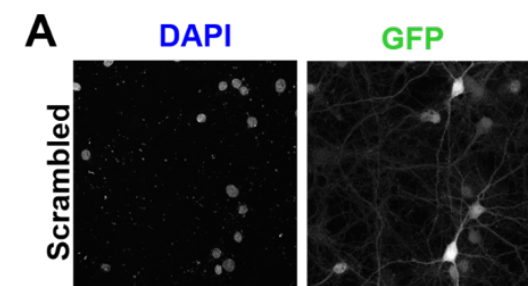

ADAR2
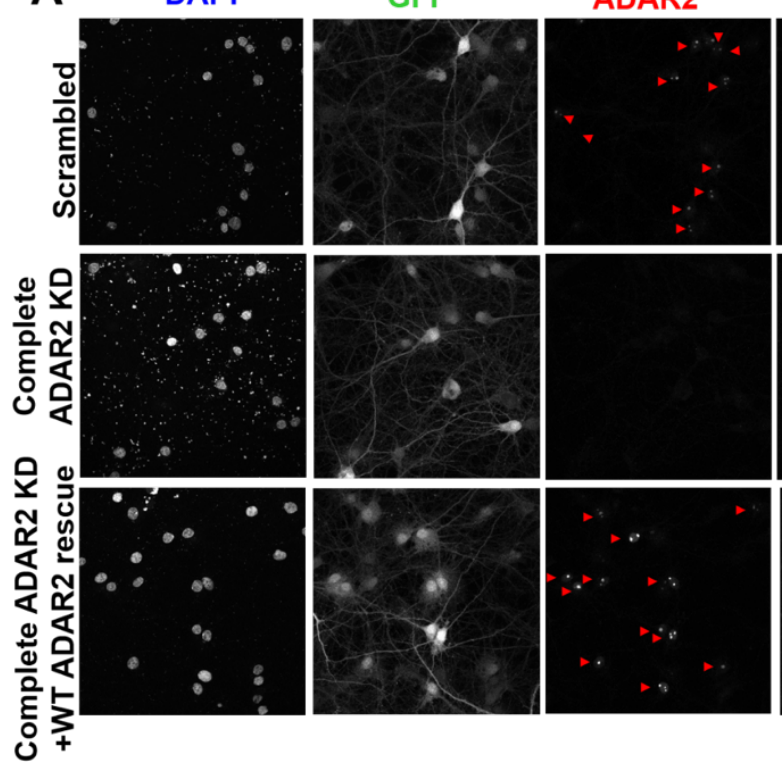

B Percentage of cells expressing ADAR2

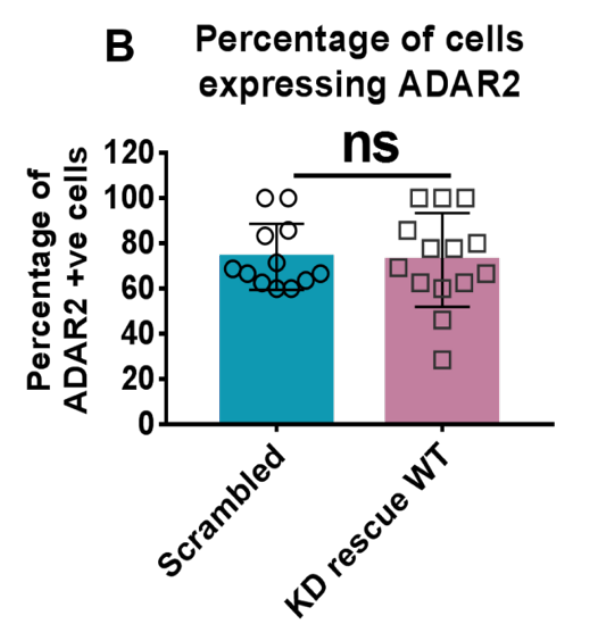

\section{Overlay}
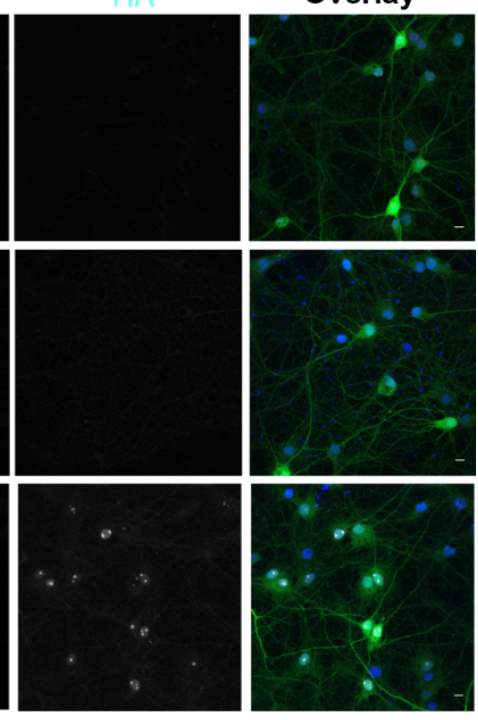

Supplementary Figure 4: Confocal imaging showing successful knockdown and rescue of endogenous ADAR2 with HA tagged WT ADAR2.

A. Representative confocal images showing nucleus imaged for DAPI (nucleus), GFP to represent the cells infected with ADAR2 knockdown, anti-ADAR2 to show successful rescue of ADAR2 in the same cells infected with the knockdown and anti-HA to show the rescues were HA tagged.

B. Quantification showing the percentage of the cells expressing the rescue is comparative to the scrambled. $\mathrm{N}=4$ independent dissections and $\mathrm{n}=12-14$ fields of view. Statistical Analysis: Unpaired t-test; ns>0.05. 

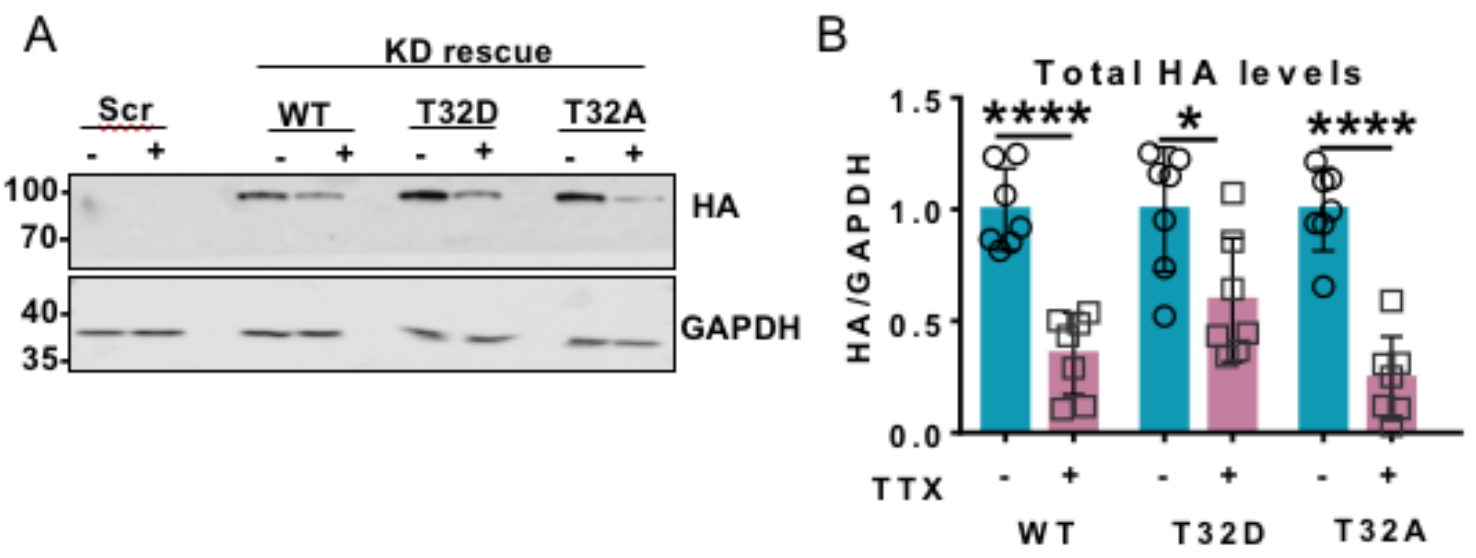

Supplementary Figure 5: HA-tagged WT, phosphonull (T32A) or phosphomimetic (T32D) ADAR2 are all equally sensitive to the $24 \mathrm{~h}$ TTX treatment.

A. Representative western blots of HA and GAPDH in cells infected with knockdown and rescued with HA tagged version of WT or T32A or T32D version of ADAR2.

B. Quantification of (A) HA signals were normalised to GAPDH. Each TTX treated condition were normalised to their respective non-treated controls. Statistical Analysis: Unpaired t-test; ${ }^{*}<0.05,{ }^{* * * *}<0.0001$. 


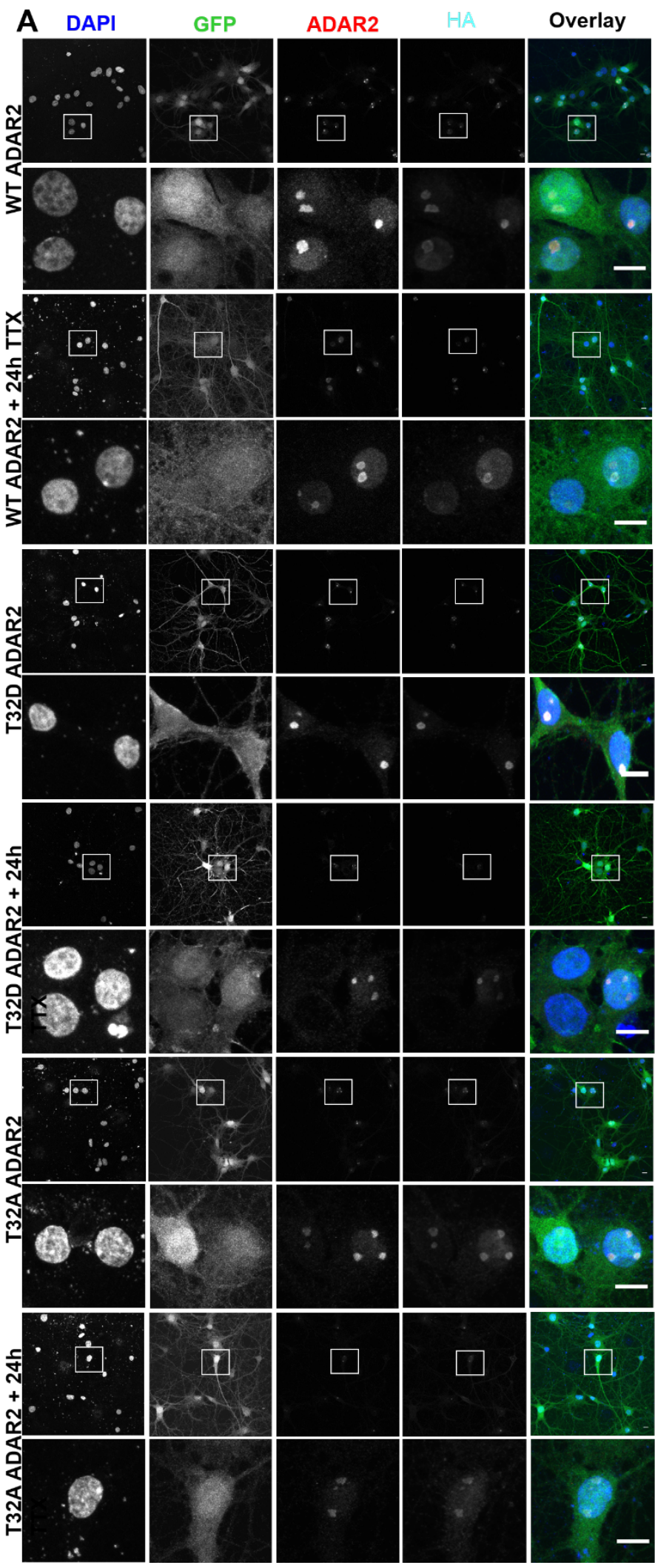

\section{B Nuclear ADAR2 levels}

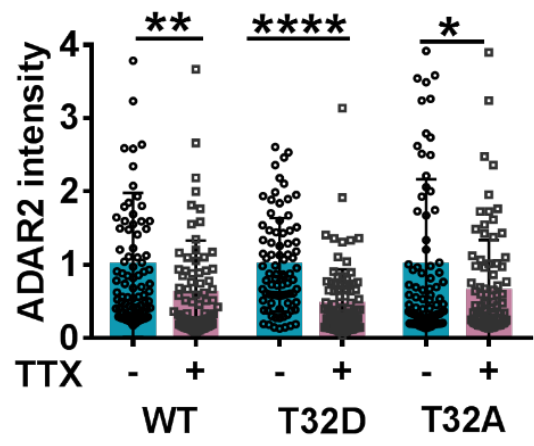

Supplementary Figure 6: Confocal imaging showing the HA tagged WT, T32A and T32D ADAR2 are localised in the nucleus and sensitive to TTX.

A. Representative images of hippocampal neurones imaged for DAPI (blue), GFP (green; infected cells), ADAR2 (red) and HA (tag) (cyan) following ADAR knockdown and rescue. The lower panels for each group show enlargements of the areas indicated by the box.

B. Quantification of ADAR2 intensity in the nucleus of the images in A). TTX treated conditions were normalised to their respective non-treated controls. Statistical Analysis: Unpaired t-test; ${ }^{*}<0.05,{ }^{* *}<0.01$, $* * * *<0.0001 . \mathrm{N}=3$ independent dissections and $n=81-92$ cells. 